Pacific Journal of Mathematics

ROUP OF AUTOMORPHISMS OF LIE GROUPS: DENSITY
PROPERTIES, BOUNDED ORBITS AND HOMOGENEOUS
SPACE OP Rederick PAUL G 


\title{
GROUPS OF AUTOMORPHISMS OF LIE GROUPS: \\ DENSITY PROPERTIES, BOUNDED ORBITS, AND HOMOGENEOUS SPACES OF FINITE VOLUME
}

\author{
F. P. Greenleaf and M. Moskowitz
}

Let $G$ be a connected Lie group and $\mathscr{A}$ an arbitrary, not necessarily connected Lie subgroup of Aut $(G)$. For a class of groups $G$ described below, the authors examine the bounded $\mathscr{C}$-orbits in $G$ (those with compact closure) and the group of bounded elements $B(G, \mathscr{A})=\{x \in G: \mathscr{A} x$ is bounded $\}$. They first show that $G$ may be split into closed, $\not$-invariant "layers" terminating at $B(G, \mathscr{A})$ whose properties insure that (i) $B(G, \mathscr{C})$ is closed, (ii) every finite, $\mathscr{A}$-invariant Borel measure must have supp $\mu \subseteq B(G, \mathscr{A})$, and (iii) if $x \in B(G, \mathscr{A})$, there is such a measure " with $x \in$ supp th. Using these results, they prove a number of density theorems of the following sort.

Theorem 1.1. Let $\mathscr{B} \subseteq \mathscr{A}$ be arbitrary, not necessarily connected Lie subgroups of Aut $(G)$ such that $\mathscr{A} / \mathscr{S}$ has finite volume (or is compact). For any $x \in G, \mathscr{B} x$ is bounded $\Leftrightarrow \quad \angle x$ is bounded.

THEOREM 1.2. Let $B \subseteq A$ be arbitrary, not necessarily connected, closed subgroups of $G$ such that $A / B$ has finite volume (or is compact). Let $\alpha \in \operatorname{Aut}(G)$ be arbitrary. Then the displacement set $\operatorname{disp}(\alpha, B)=\left\{\alpha(x) x^{-1}: x \in B\right\}$ is bounded $\Leftrightarrow \operatorname{disp}(\alpha, A)$ is bounded.

The authors prove these results for $G$ whose Levi factor is faithfully represented; there are indications that they remain true for all connected $G$. The proofs devolve to questions about faithful linear representations of certain nonconnected groups. Recent results of G. Hochschild show that $G x_{o}$ Aut $(G)$ is faithfully represented if (i) the Levi factor of $G$ is faithfully represented, and (ii) the nilradical is simply connected. In the authors' work it is crucial to know that the representation can be chosen so that $G \times I$ (if not all of $G \times_{\sigma}$ Aut $(G)$ ) is mapped to a closed subgroup of GL $(V)$; this is proved by modifying Hochschild's proofs, coupling them with methods developed by M. Goto.

Furthermore, invariant finite Borel measures play a large role: Lie theory yields information which drastically restricts the possible locations of such measures. In crucial places the converse is true: invariant measure arguments seem necessary to obtain algebraic and geometric information about the actions. 
For future reference we state the precise result concerning bounded elements $B(G, \mathscr{A})$. If a group $\mathscr{A}$ acts by homeomorphisms of a locally compact space, $\mathscr{A} \times X \rightarrow X$, the bounded orbits are defined in the obvious way. Adopting a slight modification of the terminology introduced in [5], we define a "layering" of $X$ terminating at $Y$ to be any finite sequence of closed, $\mathscr{A}$-invariant sets $X=$ $X_{0} \supseteqq X_{1} \supseteqq \cdots \supseteqq X_{m}=Y$ such that:

Each point in the $i$ th layer $X_{i} \sim X_{i-1}=L_{i}$ has a relative neighborhood $U \subseteq L_{i}$ which is moved to infinity within $L_{i}$ by a suitably chosen sequence of transforms $\left\{\alpha_{j}\right\} \cong \mathscr{C}\left(\alpha_{j}(U) \cap K=\dot{\phi}\right.$ eventually for every compact set $K \cong L_{i}$; notation: $\alpha_{j}(U) \rightarrow \infty$ in $L_{i}$ ).

Clearly then each point in $L_{i}$ has a relative neighborhood with

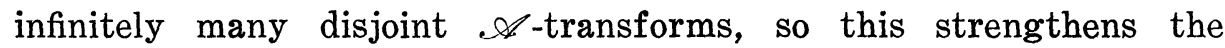
notion of layering used in [5]. Existence of a layering immediately forces all finite, $\mathscr{A}$-invariant Borel measures to be supported within the closed terminal set $Y$. The central result we wish to prove is:

THEOREM 1.3. Let $G$ be a connected Lie group whose Levi factor is faithfully represented. For any subgroup . $\cong$ Aut $(G)$,

(i) $B(G, \mathscr{L})$ is closed.

(ii) There is an $\mathscr{A}$-invariant layering from $G$ down to $B(G$,

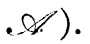

(iii) Any finite, $\mathscr{A}$-invariant Borel measure has $\operatorname{supp} \mu \subseteq B(G$, $\mathscr{A})$, and conversely if $x \in B(G, \mathscr{A})$ there is such a measure with $x \in \operatorname{supp} \mu \subseteq B(G, \mathscr{A})$.

Due to the arbitrary nature of $\mathscr{A}$ this greatly generalizes previous results of [5], [6] where $\mathscr{A}=\operatorname{Int}(G)$, and [7] where $\mathscr{A} \supseteqq \operatorname{Int}(G)$; these, in turn, took as their start the work of Tits [18] where $\mathscr{A}=$ Int $(G), G$ semisimple without compact factors. (Strictly speaking, the result are not quite related by inclusion because in the special case $\mathscr{A}=\operatorname{Int}(G)$ we were able to avoid any assumptions concerning the Levi factor.)

As we will show in $\S 4$, validity of (1.3) for some class of groups $G$ immediately leads to validity of the density Theorem 1.1 for these $G$. As for 1.3, the first step is to prove 1.3 in the case of linear actions, where $G$ is a vector group. This is done in $\S 4$; at the same time we shall point out and correct a gap in [7], closely connected with this discussion. The linear case and our discussion of faithful representations of $G \times{ }_{\sigma} \operatorname{Aut}(G)$, which are given in $\S 3$, yield 1.3 in full generality. Incidentally, the simplest property one might examine in the context of 1.1 is the question whether: $\mathscr{B} x=$ $\{x\} \Rightarrow \mathscr{A} x=\{x\}$. Counterexamples are known in which $\mathscr{B} x=\{x\}$ 
but $\mathscr{A} x$ is bounded and $\neq\{x\}$, so 1.1 seems to be the best result generally valid.

Density properties involving bounded displacement 1.2 were first examined in [18]. Partial results were given in [5] and [7], and the subject was discussed more systematically in [14]. Certain results on continuous 1-cocycles associated with a linear representation were pointed out in [14]. Their present generalizations are given in $\S 2$, and play a central role in $\S 5$, where we prove 1.2 . (They also enter into related results by one of the authors' students [17], dealing with automorphisms on minimally almost periodic groups.) They can also be used to give a very direct proof of 1.1 for linear and affine actions on a vector group, as explained in $\S 2$.

2. Preliminary results on linear and affine actions. In this section we temporarily change notation and consider a continuous linear action $G \times V \rightarrow V$ of a not necessarily connected locally compact group $G$ on a finite dimensional real vector space $V$. Letting $H$ be any closed subgroup of $G$ such that $G / H$ has finite $G$-invariant volume, we shall study the relationship between boundedness of the $H$-orbit of a subset $S \subseteq V$ and that of the $G$-orbit of $S$. We use the following notation. If $G \times X \rightarrow X$ is an action on a locally compact space $X$, let

$$
\begin{aligned}
& X_{c} \text { or } B(X, G)=\{x \in X: G x \text { is bounded (compact closure) }\} \\
& X_{\text {rix }}=\{x \in X: g \cdot x=x, \text { all } g \in G\} .
\end{aligned}
$$

In the special case when $G$ acts on itself by conjugation we use the more traditional notations $B(G)$ and $Z(G)$. If $X=V$ is a vector group and $G$ acts linearly, we write $\rho: G \rightarrow \mathrm{GL}(V)$ for the associated linear representation. Then a 1-cocycle with respect to $\rho$ is a continuous function $\phi: G \rightarrow V$ such that

$$
\dot{\phi}\left(g g^{\prime}\right)=\dot{\phi}(g)+g \cdot \dot{\phi}\left(g^{\prime}\right)=\dot{\phi}(g)+\rho_{g}\left(\dot{\phi}\left(g^{\prime}\right)\right) \text { all } g, g^{\prime} \in G \text {. }
$$

Note: These 1-cocycles correspond to the continuous affine actions $A: G \times V \rightarrow V$ related to the given $\rho$. Given such an action $A$, let $\phi(g)=A_{g}(0)$ and $\rho_{g}(v)=A_{g}(v)-\dot{\phi}(g)$. Then $A_{g}(v)=\rho_{g}(v)+\phi(g)$ for all $g, \rho$ is a uniquely determined continuous linear representation, and $\phi$ is a typical 1-cocycle for $\rho$.

Let $M(X)$ be the finite Borel measures on $X$, and $M_{c}(X)$ those with compact support; $C_{c}(X)$ and $C_{0}(X)$ are the continuous functions with compact support, or vanishing at infinity. We let $G$ act on $M(X)$ by taking

$$
\langle g \cdot \mu, f\rangle=\int_{X} L_{g} f(x) d \mu(x) \text { where } L_{g} f(x)=f\left(g^{-1} \cdot x\right) .
$$


In discussing supports of invariant measures we may always replace $\mu$ by $|\mu|$, and assume that $\mu \geqq 0$. If $f$ is a bounded function on a subset $S \subseteq X,\|f\|_{s}$ stands for the sup norm.

By dominated convergence it is easy to see that:

LEMMA 2.1. Let $G \times X \rightarrow X$ be a continuous action. If $g_{n} \rightarrow g$ in $G$ and $\mu \in M(X)$, then $g_{n} \cdot \mu \rightarrow g \cdot \mu$ pointwise on $C_{0}(X)$.

Corollary 2.2. If $D$ is a dense set in $G$ and $\mu \in M(X)$ is $D$ invariant, then $\mu$ is G-invariant.

Our next lemma requires $\mu$ to have compact support.

Lemma 2.3. Let $G$ be a subgroup of $\mathrm{GL}(V), G^{\#}$ its algebraic hull, and $\mu \in M_{c}(V)$. If $\mu$ is $G$-invariant, then it is also $G^{\sharp}$-invariant.

Proof. Let $W$ be the linear subspace spanned by supp $\mu$. Then $\mu$ is a measure on $W$ and since $\operatorname{supp} \mu$ is $G$-invariant, so is $W$. It follows that $W$ is $G^{\sharp}$-invariant. To see that $\mu$ is $G^{\sharp}$-invariant, it is enough to show that it is invariant under $G^{\#} \mid W$. By continuity of the map $g \mapsto g \mid W$ in the Zariski topology it follows that $G^{\sharp} \mid W \leqq$ $(G \mid W)^{\sharp}$. This means that we need only show that $\mu$ is invariant under $(G \mid W)^{\sharp}$; i.e., we may assume that supp $\mu$ spans $V$. Since supp $\mu$ is compact and $G$-invariant, $G$ is a bounded subgroup of GL $(V)$. But $G^{\#}$ is Euclidean closed in $\mathrm{GL}(V)$, so that $G^{\#} \supseteq G^{-}$. Since the latter group must be compact, and therefore algebraic [23], we conclude that $G^{\sharp}=G^{-}$. The result now follows from 2.2.

LEMMA 2.4. Let $\phi$ be a 1-cocycle with respect to a finite dimensional continuous representation $\rho: G \rightarrow \mathrm{GL}(V)$. Then $\dot{\phi}(G)$ is a $G$-orbit under a continuous affine action on $V$.

Proof. For $g \in G$ let $A_{g}: V \rightarrow V$ be defined by $A_{g}(v)=\rho_{g}(v)+$ $\phi(g)$. Then $A_{g}$ is an affine map on $V$, and we have a continuous affine action since

$$
\begin{aligned}
A_{g_{1}}\left(A_{g_{2}}\right)(v) & =\rho_{g_{1}}\left(\rho_{g_{2}}(v)+\dot{\phi}\left(g_{2}\right)\right)+\phi\left(g_{1}\right) \\
& =\rho_{g_{1}} \rho_{g_{2}}(v)+\phi\left(g_{1}\right)+\rho_{g_{1}}\left(\phi\left(g_{2}\right)\right) \\
& =\rho_{g_{1} g_{2}}(v)+\phi\left(g_{1} g_{2}\right)=A_{g_{1} g_{2}}(v) .
\end{aligned}
$$

Then we note that $A_{g_{1}}\left(\phi\left(g_{2}\right)\right)=\rho_{g_{1}}\left(\phi\left(g_{2}\right)\right)+\phi\left(g_{1}\right)=\phi\left(g_{1} g_{2}\right)$, so that $\phi(G)$ is $A_{G}$-stable; in particular, $A_{g}(\phi(e))=\phi(g)$, so that $\phi(G)=A_{G}(\phi(e))$.

A variant of the following lemma was stated without proof in 
[5]; for completeness we give a short proof here, since the result is used heavily later on.

Lemma 2.5. If $G \times V \rightarrow V$ is a continuous affine action and $G \cdot v_{0}$ is a bounded $G$-orbit (compact closure), then there exists a $G$ invariant finite Borel measure $\mu$ on $V$ such that $v_{0} \in \operatorname{supp} \mu \subseteq(G$. $\left.v_{0}\right)^{-}$. In particular, $\operatorname{supp} \mu=\left(G \cdot v_{0}\right)^{-}$.

Proof. As indicated in [5], Lemma 7.2, one can imbed $V$ equivariantly as a $G$-invariant hyperplane $H=V \oplus(1) \subseteq W=V \oplus \boldsymbol{R}$, where $G$ has a continuous linear action on $W$; the imbedding map $\delta: V \rightarrow H$ is a diffeomorphism onto $H$. Clearly both $W_{c}=\{w \in W$ : $G \cdot w$ is bounded $\}$ and $H$ are closed $G$-invariant subsets of $W$ and $\delta$ identifies $V_{c}$ with $H \cap W_{c}$. It suffices to show the result true for the linear action $G \times W \rightarrow W$ and any base point $w \in W_{c}$ : then for the particular base point $\delta\left(v_{0}\right)$ there would be a $G$-invariant measure $\mu$ such that $\operatorname{supp} \mu=\left(G \cdot \delta\left(v_{0}\right)\right)^{-}$. Since $\delta\left(v_{0}\right) \in H \cap W_{c}$, this support lies in $H \cap W_{c}$ and $\mu$ may be regarded as a measure on $H \cap W_{c}$; using $\delta$ to transfer it back to $V$, we are done.

Thus we assume that our action is linear. Let $K=\left(G \mid V_{c}\right)^{-}$; then $K$ is a compact subgroup of $\mathrm{GL}\left(V_{c}\right)$ and $K / \mathrm{Stab}_{K}\left(v_{0}\right)$ is a homogeneous space with a finite $K$-invariant measure. By compactness, this space is $K$-equivariantly homeomorphic to the orbit $K \cdot v_{0}$; the invariant measure gives a $K$-(hence $G$-) invariant measure on the orbit. Clearly $K \cdot v_{0}=\left(G \cdot v_{0}\right)^{-}$, so the proof is complete.

We now come to our first theorem, which depends on a recent result of S. P. Wang [19].

TheOREm 2.6. Let $G$ be a locally compact group, $\rho$ a continuous finite dimensional real representation of $G$ on $V$, $\dot{\phi}$ a 1-cocycle with respect to $\rho$, and $H$ a closed subgroup of $G$ such that $G / H$ has finite volume (or is compact). If $\dot{\phi} \mid H$ is bounded, then $\phi$ itself is bounded.

We defer the proof for a moment. Taking $\rho=i d$ (so the 1-cocycle is any continuous linear representation of $G$ ), we get the linear action case of 1.1 .

Corollary 2.7. Consider any continuous affine action $G \times V \rightarrow$ $V$ on a finite dimensional vector space. If $H$ is a closed subgroup of $G$ such that $G / H$ has finite volume (or is compact) then $V_{c, H}=$ $V_{c, G}:$ a vector has bounded $H$-orbit $\Leftrightarrow$ it has bounded G-orbit.

Proof. Exactly as in the discussion of 2.5 there is an immediate 
reduction to the case of linear actions, to which we apply 2.6.

In studying displacements $(\S 5)$ we will use 2.6 in situations where $\rho$ is nontrivial. Moreover, in $\S 4$ we will derive 2.7 from first principles by different methods-i.e., in the course of proving 1.3; these methods provide additional information which seems to be necessary in attacking the general density Theorem 1.1.

We also note that if $(G, \rho, V)$ is a system whose bounded 1-cocycles are all trivial, then any 1-cocycle known to be bounded on $H$ must in fact be trivial on both $H$ and $G$. This can be useful. For example, if $G$ acts linearly on $V$ and if we know in advance that $V_{c, G}=V_{\text {fix }}$, it follows that any vector with bounded $H$-orbit is actually $G$-fixed. In particular, if $G \times V \rightarrow V$ is a type $E$ linear action, or is a complex analytic action, or if $G$ is minimally almost periodic, this is so.

Proof of 2.6. The result is easy if $G / H$ is compact. For then $G=U H$ where $U$ is a compact set in $G$. If $g=u h$, then $\phi(g)=$ $\phi(u h)=\phi(u)+u \cdot \phi(h) € \phi(U)+U \cdot \phi(H)$; but $\phi(H)$ is bounded, and so is $\phi(G)$. Now suppose $G / H$ has finite volume. The cocycle $\phi$ is associated with an affine action $A_{g}(v)=\rho_{g}(v)+\phi(g)$, which in turn may be regarded as the restriction to the hyperplane $V \oplus(1) \subseteq W=V \oplus \boldsymbol{R}$ of the following continuous linear representation $T: G \rightarrow \mathrm{GL}(W)$,

$$
T_{g}(v, t)=\left(\rho_{g}(v)+t \phi(g), t\right) \text { all } v \in V, t \in \boldsymbol{R} .
$$

By 2.4 we have $\phi(H)=A_{H}(\phi(e))$, resp. $\phi(G)=A_{G}(\phi(e))$. Clearly these affine orbits are bounded $\Leftrightarrow$ the vector $w_{0}=(\phi(e), 1) \in W$ has bounded $T(H)$-orbit, resp. $T(G)$-orbit.

We want to show $T(G) w_{0}$ is bounded. By [19], if $G_{1}=T^{-1}\left(T(H)^{\sharp}\right)$ then $G / G_{1}$ is compact, so it suffices to show that $T\left(G_{1}\right) w_{0}$ is bounded; i.e., we may assume that $T(H) \subseteq T(G) \subseteq T(H)^{\sharp}$. By hypothesis $T(H) w_{0}$ is bounded. By 2.5 there is a finite, $T(H)$-invariant Borel measure such that $\operatorname{supp} \mu=\left(T(H) w_{0}\right)^{-}$. Since this support is compact, 2.3 insures that $\mu$ is $T(H)^{\sharp}$-invariant, hence $T(G)$-invariant. Thus $\left(T(G) w_{0}\right)^{-} \subseteq \operatorname{supp} \mu$ is compact, and $\phi$ is bounded on $G$.

Corollary 2.8. Let $\rho: G \rightarrow \mathrm{GL}(V)$ be a continuous representation of a locally compact group $G$ in a finite dimensional vector space $V$, and let $H$ be a closed subgroup such that $G / H$ has finite volume. If $\mu$ is a finite, $H$-invariant Borel measure in $M_{c}(V)$, then $G \cdot \operatorname{supp} \mu$ is compact.

Proof. By $2.3 \mu$ is invariant under $H^{\prime}=\rho^{-1}\left(\rho(H)^{\sharp}\right)$, and by [19] $G / H^{\prime}$ is compact: $G=U H^{\prime}$ where $U$ is compact. Since $\mu$ is $H^{\prime}$ - 
invariant so is $\operatorname{supp} \mu$. Hence $G \cdot \operatorname{supp} \mu=U \cdot \operatorname{supp} \mu$ is compact.

Corollary 2.9. Let $\rho: G \rightarrow \mathrm{GL}(V)$ be a continuous finite dimensional real representation of a locally compact group $G$. Let $H$ be a closed subgroup such that $G / H$ has finite volume. If a subset $S \subseteq V$ has $H \cdot S$ bounded, then $G \cdot S$ is also bounded.

Proof. Since $H \cdot S$ has compact closure, we can find a dense sequence $\left\{x_{n}=h_{n} \cdot s_{n}\right\} \subseteq(H \cdot S)^{-}$. Each orbit closure $\left(H \cdot x_{n}\right)^{-}$is associated with a finite, $H$-invariant Borel measure $\mu_{n} \geqq 0$ with precisely this set as its support, by 2.5 ; we may normalize so that $\left\|\mu_{n}\right\|=1$. Now $\mu=\sum\left\{2^{-n} \mu_{n}: n=1,2, \cdots\right\}$ is finite, $H$-invariant, and has

$$
(H \cdot S)^{-} \subseteq \operatorname{supp} \mu \subseteq\left(\bigcup_{n=1}^{\infty} \operatorname{supp} \mu_{n}\right)^{-}=\left(\bigcup_{n=1}^{\infty}\left(H \cdot x_{n}\right)^{-}\right)^{-}=(H \cdot S)^{-} .
$$

By $2.8,(G \cdot S)^{-}$is compact too.

As in the discussion of '2.7, this result extends easily to continuous affine actions. Our next corollary was also proved in [19].

CoRollary 2.10. Let $G$ and $G^{\prime}$ be locally compact groups, $H$ and $H^{\prime}$ closed subgroups, and let $\pi: G \rightarrow G^{\prime}$ be a continuous homomorphism such that $\pi(H) \cong H^{\prime}$. Suppose $G / H$ has finite $G$-invariant volume. Then

(i) There is a finite $\pi(G)$-invariant measure on $G^{\prime} / H^{\prime}$.

(ii) If $\pi(G)$ is dense in $G^{\prime}$, then $G^{\prime} / H^{\prime}$ has finite $G^{\prime}$-invariant volume.

(iii) Let $G^{-}=\pi(G)^{-}$and $H^{-}=\pi(H)^{-}$. Then $G^{-} / H^{-}$has finite $G^{-}$-invariant measure.

(iv) If $\pi$ is surjective, then $G^{\prime} / H^{-}$has finite $G^{\prime}$-invariant measure.

Proof. All groups (and their subgroups) act on the respective coset spaces by left translation. Since $\pi$ is a homomorphism, there is an action of $G$ through $\pi(G)$ on $G^{\prime} / H^{\prime}$, namely $\left(g, g^{\prime} H\right) \rightarrow \pi(g) g^{\prime} H$. Since $\pi(H) \subseteq H^{\prime}$ there is a continuous induced map from $G / H$ to $G^{\prime} / H^{\prime}$ which is $G$-equivariant, namely $p(g H)=\pi(g) H^{\prime}$. Using $p$ to transfer a $G$-invariant measure $\mu$ on $G / H$ to $\mu^{\prime}$ defined by $\mu^{\prime}(E)=$ $\varkappa^{\prime}\left(p^{-1}(E)\right)$ for Borel sets $E \subseteq G^{\prime} / H^{\prime}$, it is easy to see that $\mu^{\prime}$ is $\pi(G)$-invariant, so (i) is established. Then (ii) follows from 2.2 , and (ii) $\Rightarrow$ (iii) if we take $G^{\prime}=G^{-}, H^{\prime}=H^{-}$. Clearly (iii) $\Rightarrow$ (iv).

If $G_{1} \times X \rightarrow X$ is any continuous action on a locally compact 
space, and if $G \leqq G_{1}$ is any subgroup, it is obvious that $(G \cdot x)^{-}=$ $\left(G^{-} \cdot x\right)^{-}$for any orbit. In 1.3 this means that we may assume $\mathscr{A}$ is a closed subgroup of automorphisms, without loss of generality. In 1.1, orbit closures and their boundedness properties are unaffected if we replace $\mathscr{A}, \mathscr{B}$ by their 'closures $\mathscr{A}^{-}, \mathscr{B}^{-}$in the automorphism group. Since the finite volume condition passes from $\mathscr{A} \mid \mathscr{B}$ to $\mathscr{A}^{-} / \mathscr{B}^{-}$by 2.10 , we may assume from the start that $\mathscr{A}, \mathscr{B}$ are closed groups of automorphisms in discussing 1.1.

We end this section with some results on boundedness of conjugacy classes in linear Lie groups. The method will reappear later on.

THEOREM 2.11. Let $G$ be a closed linear Lie group (not necessarily connected) and $H$ a closed subgroup such that $G / H$ has finite volume. If the $H$-conjugacy class $C_{H}(g)=\left\{h g h^{-1}: h \in H\right\}$ of an element $g \in G$ has compact closure in $G$, then so does the G-conjugacy class $C_{G}(g)$. In fact, if $S$ is any subset of $G$, then $C_{H}(S)$ has compact closure in $G \Leftrightarrow C_{G}(S)$ does.

Proof. By imbedding $\mathrm{GL}(V)$ in $\mathrm{SL}(V \oplus \boldsymbol{R})$ in the obvious way, we may pass to a situation in which $G, H$ are closed subsets in the space of all linear operators $W=\operatorname{End}(V)$. Now let $g \in G$ act on $W$ by similarity transforms: $X \rightarrow g X g^{-1}$. This linear action $G \times W \rightarrow$ $W$ when restricted to $G \leqq W$ yields the action of $G$ on itself by conjugation. Let $g \in G$ and assume $C_{H}(g)$ is bounded in $G(\Leftrightarrow$ bounded in $W)$. By 2.7, $C_{G}(g)=\left\{x g x^{-1}: x \in G\right\}=\{x \cdot g: x \in G\}$ is bounded in $W$, and since $G$ is closed in $W$, it is a bounded set in $G$ itself. For the action on a set $S \subseteq G$, use the same argument as in 2.9.

REMARK. For closed linear Lie groups, we get the following relations between the bounded elements $B(H)=B(H$, Int $(H))$ in $H$ and the bounded elements $B(G)$ in $G$, when $G / H$ has finite volume:

(i) $B(H)=H \cap B(G)$.

(ii) If $B(G)$ is trivial: $B(G)=Z(G)$, then $B(H)=Z(H)$.

We have $B(G)=Z(G)$ in the following particular situations: if $G / R$ has no compact factors and the radical $R$ is simply connected of type $E$, or if $G$ is a complex analytic group, or if $G$ is minimally almost periodic [14].

3. Some theorems on faithful representations. The main purpose of this section is to prove theorems concerning faithful representations of certain not necessarily connected Lie groups. For the most part the methods make use of recent results of Hochschild [10], which depend on extensive earlier work by Hochschild and 
Mostow, and of the work of Goto [3], [4] on faithful representations with closed range. We are grateful to Prof. Hochschild for communicating the main arguments of [10] to us, and for other helpful comments. Some of the details from [10] are included below for the sake of completeness; in other places, arguments from [10] are presented in substantially revised form due to our need to construct faithful representations with closed range in GL $(V)$. In this section we shall write $\mathscr{A}(G)=\operatorname{Aut}(G)$ and $. \mathscr{T}(G)=\operatorname{Int}(G)$, for brevity.

The following preliminary results show that the connected Lie groups $G$ we have in mind (Levi factor faithfully represented, simply connected nilradical) have faithful representations with certain additional properties.

Lemma 3.1. Let $G$ be a connected Lie group, $R$ its radical and $S$ a Levi factor. If $S$ has a faithful representation then the semidirect product $R \times{ }_{0} S$, where $S$ acts by conjugation on $R$, is a finite covering of $G$. Moreover, if $R$ is simply connected, then $G \cong R \times{ }_{\text {"I }} S$.

Direct calculations show that $\pi(r, s)=r \cdot s$ is a homomorphism of $G \times{ }_{0} S$ to $G$; it is surjective since $G=R \cdot S$, hence is an open mapping. Its kernel $F$ is closed, normal in $R \times{ }_{0} S$; clearly $F=\left\{\left(x^{-1}, x\right)\right.$ : $x \in R \cap S\}$ and $R \cap S$ is discrete in the Lie topology of $S$, from which we see that $F$ is discrete (hence central) in $R \times_{0} S$. Thus $\pi$ is a covering homomorphism. Since $S$ is faithfully represented it has finite center, hence $S \cap R$ (and $F$ ) are finite If $R$ is simply connected, it has no proper compact subgroups, so $S \cap R=(e)$ and $F$ is trivial.

LemMA 3.2. Let $G$ be a subgroup of GL(V), $F$ a normal subgroup compact in the relative topology (for example, a finite central subgroup) and let $U$ be a subset of $G$ consisting of unipotent elements. Then there exists a finite dimensional representation $\rho: G \rightarrow$ $\mathrm{GL}(W)$ such that

(i) $\operatorname{Ker} \rho=F$

(ii) $\rho(U)$ acts on $W$ by unipotent operators.

Proof. Let $G^{\sharp}$ be the algebraic hull of $G$ in GL $(V)$. Then $F$ is a compact and therefore algebraic subgroup of $G^{*}$ [23]. Since $F$ is normalized by $G, G$ is Zariski dense in $G^{*}$, and $F$ is algebraic, $F$ is normal in $G^{*}$. By a theorem of Chevalley [1] there exists a rational representation $\rho$ of $G^{\#}$ such that $\operatorname{Ker} \rho=F$. Since $\rho$ is rational and $U$ consists of unipotent elements so does $\rho(U)$. Clearly then the restriction of $\rho$ to $G$ satisfies (i) and (ii). 
Lemma 3.3. Let $G$ be any 2 nd countable locally compact group, $\sigma$ and $\tau$ representations of $G$ on $V_{\sigma}$ and $V_{\tau}$ respectively. If $\sigma$ is faithful and has closed range then $\sigma \oplus \tau$ is also faithful and has closed range.

Proof. For $g \in G$,

$$
(\sigma \oplus \tau)(g)=\left[\begin{array}{cc}
\sigma(g) & 0 \\
0 & \tau(g)
\end{array}\right] .
$$

If $(\sigma \oplus \tau)\left(g_{n}\right) \rightarrow T$ in GL $\left(V_{\sigma} \oplus V_{\tau}\right)$ then clearly $T$ is block diagonal, consisting of blocks $A, B$; thus $\sigma\left(g_{n}\right) \rightarrow A$ and $\tau\left(g_{n}\right) \rightarrow B$. By the open mapping theorem applied to $\sigma$ we know $A=\sigma(g)$ for some $g \in G$, and $g_{n} \rightarrow g$. By continuity, $\tau\left(g_{n}\right) \rightarrow \tau(g)$, so $B=\tau(g)$ and $T=$ $(\sigma \oplus \tau)(g)$ as required to show $\sigma \oplus \tau$ has closed range. It is clearly faithful.

Proposition 3.4. Let $G$ be a connected Lie group with faithfully represented Levi factor $S$, and simply connected nilradical $N$. Then there exists a faithful representation $\rho: G \rightarrow \mathrm{GL}(V)$ which maps $N$ to unipotent operators and $\rho(G)$ is closed in GL $(V)$.

Proof. We first prove the result except for closedness of $\rho(G)$. Let $G=R \cdot S$ be the Levi decomposition. Since $S$ is faithfully represented, we know by 3.1 that $\pi: R \times{ }_{\theta} S \rightarrow G$ is a finite covering. As a covering map we have $\pi\left(N\left(R \times_{0} S\right)\right)=N(G)=N$. Suppose we had a faithful representation $\sigma$ of $R \times_{0} S$ which was unipotent on $N\left(R \times{ }_{0} S\right)$. Then since $\operatorname{Ker} \pi=F$ is a finite central subgroup, 3.2 would give a representation $\rho$ of $R \times{ }_{0} S \rightarrow \mathrm{GL}(V)$ under which $N\left(R \times{ }_{0} S\right)$ acts unipotently and $\operatorname{Ker} \rho=F$. Therefore $\rho$ induces a faithful representation of $G$ under which $N$ acts unipotently. We may therefore assume $G=R \times{ }_{0} S$.

Case 1. $S=(e)$, that is $G=R$ is solvable. Here $[R, R]^{-} \leqq N$ so $R / N$ is abelian. Thus $R / N=E \times T$, the direct product of a vector group and a torus. If $\pi: R \rightarrow R / N$ denotes the projection then $\pi^{-1}(E)$ is a connected simply connected normal analytic subgroup of $R$ containing $N$ and $R / \pi^{-1}(E) \cong T$. By Iwasawa's splitting theorem, $R=\pi^{-1}(E) \times{ }_{\theta} T$. Since $\pi^{-1}(E)$ is simply connected, there exists, by Hochschild-Mostow [9], a faithful representation $\rho_{1}$ of $\pi^{-1}(E)$ on $V_{1}$ which is unipotent on the nilradical of $\pi^{-1}(E)$, and in particular on $N$. Now $\left[\pi^{-1}(E), R\right] \subseteq[R, R] \subseteq N$, so $\rho_{1}$ acts unipotently on $\left[\pi^{-1}(E), R\right]$. By Theorem 2.2, p. 215, of [9] there exists a representation $\sigma: R \rightarrow \mathrm{GL}(W)$ such that $V_{1} \subseteq W$ via a $\pi^{-1}(E)$ - 
equivariant imbedding, and $\sigma$ is unipotent whenever $\rho_{1}$ is unipotent. In particular $\sigma$ is unipotent on $N$. Now $R / \pi^{-1}(E)$ is compact. By the Peter-Weyl theorem there exists a continuous faithful representation of $R / \pi^{-1}(E)$. Its lift back $\tau$ to $R$ is a representation of $R$ with $\operatorname{Ker} \tau=\pi^{-1}(E)$. Then $\gamma=\sigma \oplus \tau$ is easily seen to be faithful. Moreover $\gamma$ is unipotent on $N$ since on $\pi^{-1}(E), \gamma=\sigma \oplus I$, and $\sigma$ is unipotent on $N$.

Case 2. $G=R \times{ }_{\theta} S$. By Case 1 there exists a faithful representation $\gamma$ of $R$ on $W$ which is unipotent on $N$. Taking differentials we see that by Theorem 3.2, p. 128 of [9], $\gamma[G, R]$ is unipotent. By 2.2, p. 215 of [9], $\gamma$ extends $R$-equivariantly to $\rho$ on $V$ such that $\rho$ is unipotent whenever $\gamma$ is unipotent. In particular, $\rho$ is unipotent on $N$.

By hypothesis $S$ has a faithful representation. Let $\tau$ be its lift to $R \times{ }_{\theta} S$. Then $\operatorname{Ker} \tau=R$. Now $\rho \oplus \tau$ is a representation of $R \times{ }_{0} S$ which is faithful for if $(\rho \oplus \tau)(g)=1$ then $g \in R$ and $\rho(g)=1$, therefore $\gamma(g)=1$ and so $g=1$. Clearly $\rho \oplus \tau$ is unipotent on $N$ since on $R$ it equals $\rho \oplus I$ and $\rho$ is unipotent on $N$.

Now there exists a faithful representation $\rho$ of $G$ on $V$ which is unipotent on $N$; but $\rho(G)$ may not have closed range. To deal with this condition we apply the techniques of $M$. Goto in [3] and [4]. As in Case 1 discussed above, $R=M \times_{0} T$ where $T$ is a maximal compact subgroup of $R$ and $M=\pi^{-1}(E)$ where $\pi: R \rightarrow R / N$ is the canonical projection. Here $M$ is a closed normal simply connected subgroup of $R$ and $T[G, G]$ is a closed normal subgroup of $G$. (The fact that $[G, G]$ is closed in $G$ follows from the existence of a faithful representation $\rho$ (Theorem 4.5 of [3]), and the normality of the various groups from the fact that they contain the respective derived groups.) Also since $G$ is faithfully represented we have by [4] $G=S T M$ and $S T \cap M=(e)$. Now the Levi decomposition of $[\mathfrak{g}, \mathfrak{g}]$ is $[\mathfrak{g}, \mathfrak{g}]=[\mathfrak{g}, \mathfrak{x}] \oplus \mathfrak{g}$, so that $S T[G, R] \subseteq[G, G] T[G, R]=T[G, G]=$ $[G, G] T$. Locally the latter is $[G, R] S T=S T[G, R]$. Since we are dealing with analytic groups we have

$$
S T[G, R]=T[G, G] .
$$

In particular, $S T[G, R]$ is a subgroup although $S T$ need not be. Now $[G, R] \subseteq M$. In fact $[G, R] \subseteq N$ since it is represented unipotently under any representation of $G$ and in particular the adjoint representation. Hence

$$
G=S T M=T[G, G] M .
$$

Next we observe that $M \cap T[G, G] \subseteq[G, R]$ for if $m \in M \cap T[G, G]$ 
then $m=$ sty where $s \in S, t \in T$ and $y \in[G, R]$ but $m y^{-1} \in M \cap S T=$ (e) so $m=y \in[G, R]$. Since $[G, R]$ is clearly contained in $M \cap T[G$, $G]$ we have

$$
[G, R]=M \cap T[G, G]
$$

But then

$$
G / T[G, G]=T[G, G] M / T[G, G] \cong M / M \cap T[G, G]=M /[G, R] .
$$

Since $M$ is simply connected and $[G, R]$ is connected $M /[G, R]$ is simply connected. It is also abelian since $T[G, G] \supseteqq[G, G]$. Thus $G / T[G, G]$ is a vector group. Thus, there exists a unipotent representation $\sigma$ of $G$ whose kernel is $T[G, G]$. Since $\rho$ is faithful so is $\rho \oplus \sigma$. Since $\rho$ is unipotent on $N$ and $\sigma$ unipotent on $G, \rho \oplus \sigma$ is unipotent on $N$. If we knew $\rho \oplus \sigma(M)$ were closed we would be done; since $R=M \times_{\theta} T$ we would get $\rho \oplus \sigma(R)=\rho \oplus \sigma(M) \cdot \rho \oplus \sigma(T)$ where $\rho \oplus \sigma(T)$ is compact since $T$ is. Finally, since $\rho \oplus \sigma$ is faithful and $R$ has closed range under it so must $G$ by Theorem 2 of Goto [3].

To see $\rho \oplus \sigma(M)$ is closed it is sufficient by [3] or [9] to show $\rho \oplus \sigma(\exp \boldsymbol{R} X)$ is closed for each 1-parameter subgroup of $M$. If $X \in \mathfrak{m} \cap(\mathrm{t} \oplus[\mathfrak{g}, \mathfrak{g}])$ then $\exp t X \in M \cap T[G, G]=[G, R]$ for all $t$; but any representation of $G$ is unipotent on $[G, R]$, thus $(\rho \oplus \sigma)(\exp t X)$ is unipotent for all $t$. As a unipotent analytic linear group it is closed. If $X \in \mathfrak{m} \sim \mathrm{t} \oplus[\mathfrak{g}, \mathfrak{g}]$, then $t X \notin t \oplus[\mathfrak{g}, \mathfrak{g}]$ for any $t \neq 0$. This means that $\sigma \mid \exp \boldsymbol{R} X$ is locally faithful. But since $\sigma$ is unipotent on $G$ and in particular on $\exp (\boldsymbol{R} X), \sigma \mid \exp (\boldsymbol{R} X)$ is faithful and the 1-parameter group $\sigma(\exp R X)$ is closed. Then so is $(\sigma \oplus \tau)(\exp$ $\boldsymbol{R} X)$ by 3.3. This completes the proof of 3.4 .

In what follows Aut (g) will denote the automorphisms of the Lie algebra $g$ and Aut $(\mathfrak{g})_{0}$ its Euclidean identity component. The next two lemmas, outlined in [10], are given here for the sake of completeness.

LEMma 3.5. Let $G$ be a connected Lie group, $R$ the radical, $N$ the nilradical, and let $\mathfrak{g}, \mathfrak{x}, \mathfrak{n}$ be the Lie algebras. Then each $\alpha \in$ Aut $(\mathfrak{g})_{0}$ maps $\mathfrak{x}$ into $\mathfrak{n}$. If $d$ : Aut $(G) \rightarrow$ Aut $(\mathfrak{g})$ is the differential map (an injective analytic homomorphism), let $\mathscr{A}^{\prime}=d^{-1}\left(\operatorname{Aut}(\mathrm{g})_{0}\right)$. Then $\mathscr{A}^{\prime}$ is a normal subgroup of finite index in Aut $(G)$ which contains Aut $(G)_{0} \supseteqq \operatorname{Int}(G)$ and induces trivial action on $R / N$.

Proof. Aut (g) is algebraic and Aut $(\mathfrak{g})_{0}$ is of finite index by [22]. The derivations Der $(\mathfrak{g})$ constitute the Lie algebra of Aut $(\mathfrak{g})_{0}$. By a well known theorem (see Jacobson, Lie Algebras, §3.6) $D(\mathfrak{x}) \subseteq$ 
$\varkappa$ for an $D \in \operatorname{Der}(\mathrm{g})$; therefore, all automorphisms $\alpha=e^{D}$ map $\mathfrak{x}$ into $\mathfrak{n}$, as do all the $\alpha \in \operatorname{Aut}(\mathfrak{g})_{0}$. Now $\mathscr{A}^{\prime}$ is clearly closed, normal, of finite index in $\operatorname{Aut}(G)$, so $\mathscr{A}^{\prime} \supseteqq \operatorname{Aut}(G)_{0} \supseteqq \operatorname{Int}(G)$; clearly $\mathscr{A}^{\prime}$ induces trivial action on $R / N$.

Definition. For a connected lie group $G$ let $\mathscr{T}=\{\tau \in \mathscr{A}(G)$ : $\tau$ leaves the Levi factor $S$ pointwise fixed\}, and let $\mathscr{T}^{\prime}=\mathscr{A}^{\prime} \cap \mathscr{T}$. If $\alpha \in \mathscr{A}(G)$ we have $\alpha \alpha_{g} \alpha^{-1}=\alpha_{\alpha(g)}$, so $\mathscr{Y}(G)$ is normal in $\mathscr{A}(G)$ and in $\mathscr{A}^{\prime}$; hence $\mathscr{T}^{\prime} \mathscr{J}(G)$ is a subgroup of $\mathscr{\mathscr { P } ^ { \prime }}$ and $\mathscr{Y} \mathscr{Y}(G)$ is a subgroup of $\mathscr{A}(G)$.

Lemma 3.6. $\mathscr{Y}^{\prime} . \mathcal{T}(G)$ is a closed, normal subgroup of finite index in $\mathscr{A}(G)$ which contains $\mathscr{A}(G)_{0}$.

Proof. Let $\tau \in \mathscr{T}$ and $\alpha \in \mathscr{A}(G)$. Then $\alpha^{-1}(S)$ is a maximal semisimple analytic subgroup and therefore is a Levi factor. By Malcev's theorem, there is a $g \in G$ with $\alpha_{g} \alpha^{-1}(S)=S$, hence also $\alpha \alpha_{q}^{-1}(S)=S$. Now

$$
\begin{gathered}
\alpha_{g} \alpha^{-1} \tau \alpha \alpha_{g}^{-1}(s)=\alpha_{g} \alpha^{-1} \tau\left(s^{\prime}\right)=\alpha_{g} \alpha^{-1}\left(s^{\prime}\right) \\
=\alpha_{g} \alpha^{-1} \alpha \alpha_{g}^{-1}(s)=s
\end{gathered}
$$

for $s \in S, \quad$ so $\alpha_{g} \alpha^{-1} \mathscr{\mathscr { T }} \alpha \alpha_{g}^{-1} \subseteq \mathscr{T}$ and $\alpha^{-1} \mathscr{\mathscr { V }} \alpha \cong \alpha_{g}^{-1} \mathscr{T} \alpha_{g}$. Conversely, $\tau \alpha_{g} \alpha^{-1}(s)=\alpha_{g} \alpha^{-1}(s) \Rightarrow \alpha \alpha_{g}^{-1} \tau \alpha_{g} \alpha^{-1}(s)=s$, so that $\alpha_{g}^{-1} \mathscr{S}^{-} \alpha_{g}=$ $\alpha^{-1} \cdot \mathscr{T} \alpha$. (Both inclusions were needed since $\mathscr{T}$ need not be connected.) Now if $\alpha \in \mathscr{A}(G)$, there is a $g \in G$ such that $\alpha \mathscr{F} \mathscr{J}(G) \alpha^{-1}=$

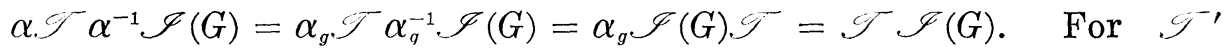
we get $\alpha \mathscr{T}^{\prime} \mathscr{I}(G) \alpha^{-1} \cong \mathscr{J} \mathscr{J}(G) \cap \mathscr{A}^{\prime}$ since $\mathscr{A}^{\prime} \supseteqq \mathscr{J}(G)$, and conversely $\mathscr{T} \mathscr{I}(G) \cap \mathscr{A}^{\prime} \subseteq \mathscr{T}^{\prime} \mathscr{I}(G)$, for if $\tau \alpha_{g} \in \mathscr{A}^{\prime}$, then $\tau \in \mathscr{A}^{\prime} \alpha_{g}^{-1} \subseteq$ . $\mathscr{C}^{\prime}$ and $\tau \in \mathscr{T}^{\prime}$. Thus $\mathscr{F}^{\prime} \mathscr{I}(G)$ is normal in $\mathscr{A}(G)$.

To see that $\mathscr{T}^{\prime} \mathscr{I}(G)$ has finite index in $\mathscr{A}(G)$ it suffices to show that $\mathscr{F} \mathscr{F}(G)$ has finite index, since $\left[\mathscr{T}: \mathscr{T}^{\prime}\right]<\infty$. For each $\alpha \in A(G)$ choose a $g \in G$ as above (nonunique, depending on $\alpha$ ) so $\alpha_{g} \alpha \mid S \in \mathscr{A}(S)$. Let $(\sim)$ be the map sending $\mathscr{A}(S) \rightarrow \mathscr{A}(S) / \mathscr{I}(S)$. Then $\alpha \mapsto\left(\alpha_{g} \alpha \mid S\right)^{\sim}$ is a map from $\mathscr{A}(G)$ to the finite group $\mathscr{A}(S) /$ $\mathcal{F}(S)$. When do $\alpha$ and $\beta$ have the same image? Same image $\Rightarrow$ $\alpha_{g} \alpha\left|S=\alpha_{s_{0}} \circ\left(\alpha_{h} \beta\right)\right| S$ for some $s_{0} \in S \Rightarrow g \alpha(s) g^{-1}=s_{0} h \beta(s) h^{-1} s_{0}^{-1}$ for all $s \in S \Rightarrow \alpha(s)=g^{-1} s_{0} h \beta(s)\left(g^{-1} s_{0} h\right)^{-1}=\alpha_{x} \beta(s)$ all $s \in S$, for some $x \in G \Rightarrow$ (since $\mathscr{I}(G)$ is normal in $\mathscr{A}(G)$ ) $\alpha(s)=\beta \alpha_{y}(s)$ all $s \in S$, for some $y \in$ $G \Rightarrow \alpha_{y}^{-1} \beta^{-1} \alpha \in \mathscr{T}$ or $\beta^{-1} \alpha \in \mathscr{I}(G) \mathscr{T}=\mathscr{T} \mathscr{I}(G)$. Hence $\beta, \alpha$ in

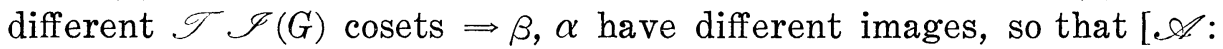
$\mathscr{T} \mathscr{I}(G)]<\infty$.

Finally, we show $\mathscr{I}^{\prime} \mathscr{I}(G) \supseteqq \mathscr{A}(G)_{0}$. Let $\alpha \in \mathscr{A}(G)_{0}$; then $\alpha=$ $\alpha_{1} \cdot \cdots \cdot \alpha_{n}$ where each $\alpha_{k}$ lies on a 1-parameter group. Since 
$\mathscr{T}^{\prime} \mathscr{I}(G)$ is a group we may assume $\alpha$ itself lies on a 1-parameter group $\alpha(t)$ in $\mathscr{A}(G)_{0} \leqq \mathscr{A}^{\prime}$. Choose $g \in G$ so that $\alpha_{g} \alpha \mid S \in \mathscr{A}(S)$. Since $n=[\mathscr{A}(S): \mathscr{I}(S)]<\infty, \quad\left(\alpha_{g} \alpha \mid S\right)^{n}=\left(\alpha_{g} \alpha\right)^{n} \mid S \in \mathscr{J}(S)$. Due to normality of $\mathscr{J}(G)$, we may write $\left(\alpha_{g} \alpha\right)^{n}=\alpha^{n} \alpha_{g^{\prime}}=\alpha_{g^{\prime}}, \alpha^{n}$ for suitable $g^{\prime}, g^{\prime \prime} \in G$. Thus there is an $x \in G$ such that $\alpha_{x} \alpha^{n}\left|S=\alpha_{s}\right| S$ for some $s \in S$ ( $x$ and $s$ depend on $\alpha$ ). Now consider the 1-parameter group $\alpha(t)$. Choose $g(t)$ and $s(t)$ so that $\alpha_{g(t)} \alpha(t)^{n} \mid S=\alpha_{s(t)}$ where $g(t), s(t)$ are suitably chosen points in $G, S$; then $\alpha_{s(t)}^{-1} \alpha_{g(t)} \alpha(t)^{n}|S=i d| S$, or $\alpha_{h(t)} \alpha(n t)|S=i d| S$ for suitable $h(t) \in G$. This means that $\alpha_{h(t)} \alpha(n t) \in \mathscr{T}$ and since everything is in $\mathscr{A}^{\prime}$, $\alpha_{h(t)} \alpha(n t) \in \mathscr{T}^{\prime}$, so that $\alpha(n t) \in \mathscr{T}^{\prime} \mathscr{I}(G)$ for all $t \in \boldsymbol{R}$. Choose $t$ so that $n t=1$; then we get $\alpha \in \mathscr{T}^{\prime} \mathscr{J}(G)$.

We now come to the main result of $\S 3$, namely 3.7. This result is an amplification and strengthening of the theorem of G. Hochschild in [10]. It also represents an extension of the results of Goto in that $\rho(G \times(I))$ is closed.

THEOREM 3.7. Let $G$ be a real (or complex) connected Lie group whose Levi factor is faithfully represented. (In the complex case this is automatic) and whose nilradical $N$ is simply connected. Then the semidirect product $G \times{ }_{0} \mathscr{C}(G)$ has a faithful finite dimensional real (or complex) analytic representation $\rho$ with the property that $\rho\left(G \times{ }_{\theta}(I)\right.$ is closed.

Note. In particular, by the open mapping theorem, $\rho$ is a topological isomorphism of $G \times{ }_{\theta} \mathscr{A}(G)$ with its image, and $\rho(G \times(I))$ is closed in $\rho\left(G \times{ }_{\theta} \mathscr{A}(G)\right)$ and hence in the general linear group. It is in this form that we will apply 3.7.

Proof. By 3.4 choose a faithful finite dimensional representation $\rho$ of $G$ which is unipotent on $N$ such that $\rho(G)$ is closed. Let $R_{\rho}$ denote the space of representative functions associated with $\rho . R_{\rho}$ is a finite dimensional space of analytic functions on $G$ which is stable under left and right translation. In particular, $R_{\rho^{\circ}} \mathscr{J}(G)=$ $R_{\rho}$, and

$$
R_{\rho} \circ \mathscr{T}^{\prime}(G) \mathscr{I}(G)=R_{\rho} \circ I(G) \mathscr{T}^{\prime}=R_{\rho} \circ \mathscr{T}^{\prime} .
$$

Now $R_{\rho} \circ \mathscr{T}^{\prime}$ has finite dimensional span. To see this we apply Lemma 2.1 , p. 213 of [9]. We need to verify that $\rho\left(\tau(x) x^{-1}\right)$ is unipotent for all $\tau \in \mathscr{T}^{\prime}$ and $x \in R(G)$, and that $\rho\left(\tau(x) x^{-1}\right)=1$ for all $\tau \in \mathscr{T}^{\prime}$ and $x \in S$. The latter point is obvious by definition of $\mathscr{T}$. Since $\mathscr{J}^{\prime} \subseteq$ $\mathscr{A}^{\prime}$ and $\mathscr{A}^{\prime}$ acts trivially on $R / N, \tau(x) x^{-1} \in N$ for all $\tau \in \mathscr{T}^{\prime}$ and $x \in R$; however since $\rho$ is unipotent on $N$ the result follows. Let 
$\mathscr{B}=\mathscr{S}^{\prime} \mathscr{J}(G)$ and let l.s. $\left\{R_{\rho} \circ \mathscr{B}\right\}=W$. Then $\mathscr{B}$ is an open normal subgroup of $\mathscr{A}(G)$ of finite index and $W$ is a finite dimensional space of analytic functions on $G$ stable under $\mathscr{B}$. It is also stable under right translations since

$$
R_{x}(f \circ \beta)(y)=f(\beta(y x))=\left(R_{\beta(x)} f\right)(\beta y) .
$$

and $R_{\rho}$ is stable under right translations.

Form the semidirect product $G \times{ }_{\theta} \mathscr{B}$ and let it act on $W$ so that $[(x, \beta) w](y)=w\left(\beta^{-1}(y x)\right)=R_{x}\left(w^{\beta}\right)(y)$ for $x, y \in G$ and $\beta \in \mathscr{B}$; here $w^{\beta}(x)=w\left(\beta^{-1} x\right)$. These are linear operators on $W$, and it is easily seen that we get a continuous linear action of the semidirect product on $W$. This representation, which we call $\sigma$, is faithful on $G \times(I)$ : if $(x, I) w=w$ for all $w \in W$, then in particular for the matrix coefficients of $\rho$ we get $\rho_{i j}(y x)=\rho_{i j}(y)$ for all $y \in G$, hence $\rho(y x)=\rho(y)$ for all $y$, and taking $y=e$ we get $\rho(x)=I$ which implies $x=e$ since $\rho$ is faithful.

We now show that the image $\sigma(G)$ of $G \times(I)$ under this representation is closed in GL $(W)$. Suppose $\sigma_{\left(x_{n}, I\right)} \rightarrow T \in \mathrm{GL}(W)$; then for the matrix coefficients $\rho_{i j}$ we have $\sigma\left(x_{n}, I\right) \rho_{i j} \rightarrow T\left(\rho_{i j}\right)$ for all $i, j$. But $\sigma(x, I) \rho_{i j}=R_{x} \rho_{i j}$, an element of $R_{\rho}$, for all $x \in G$; thus, $T\left(\rho_{i j}\right) \in$ $R_{\rho}$ and $T$ leaves $R_{\rho}$ stable. Now consider the action $G \times R_{\rho} \rightarrow R_{\rho}$ by right translation. This representation of $G$ is faithful since $\rho$ is. If $G$ had closed range under it, the open mapping theorem would insure that there exists an $x \in G$ such that $T\left|R_{\rho}=R_{x}\right| R_{\rho}$ and $x_{x} \rightarrow x$ in $G$. But then by continuity, $\sigma\left(x_{n}, I\right) \rightarrow \sigma(x, I)$ so $T=\sigma(x, I)$ and we would be done.

To see that the image of $G$ in $\mathrm{GL}\left(R_{\rho}\right)$ is closed, suppose $T \in$ GI $\left(R_{\rho}\right)$ and $R_{x_{n}} \rightarrow T$ for some sequence of $x_{n} \in G$. Then $R_{x_{n}}\left(\rho_{i j}\right) \rightarrow$ $T\left(\rho_{\imath j}\right)$ pointwise for each $i j$. Also, $R_{x_{n}}^{-1}=R_{x_{n-1}} \rightarrow T^{-1}$ in $\operatorname{GI}\left(R_{\rho}\right)$ so $R_{x_{n}}^{-1}\left(\rho_{i j}\right) \rightarrow T^{-1}\left(\rho_{i j}\right)$ pointwise for each $i j$. Evaluation at $g=e$ yields $\rho_{i j}\left(x_{n}\right) \rightarrow t_{i j}$ and $\rho_{i j}\left(x_{n}^{-1}\right) \rightarrow s_{i j}$ where $t_{i j}=T \rho_{i j}(e)$ and $s_{i j}=T^{-1} \rho_{i j}(e)$. Since this holds for all $i, j$ we get $\rho\left(x_{n}\right) \rightarrow t$ and $\rho\left(x_{n}^{-1}\right) \rightarrow s$ where $t=\left(t_{i j}\right)$ and $s=\left(s_{i j}\right)$ are square matrices. In particular, $\rho\left(x_{n}\right) \rho\left(x_{n}^{-1}\right)=$ $\rho\left(x_{n} x_{n}^{-1}\right)=e \rightarrow t s$ so that $t$ is invertible and $\rho\left(x_{n}\right) \rightarrow t$ in GI $\left(V_{\rho}\right)$. Since $\rho$ has closed range and is faithful the open mapping theorem furnishes us with an $x \in G$ so that $x_{n} \rightarrow x$. Hence $R_{x_{n}} \rightarrow R_{x}$ so $T=R_{x}$.

LeMma 3.8. Let $\rho: H \rightarrow \mathrm{GL}(V)$ be a continuous faithful representation, let $G \supseteqq H$ with finite index, and define $\tau=\operatorname{Ind}(H \uparrow G, \rho)$. If $L \subseteq H$ is any subgroup on which $\rho$ is faithful with closed range $\rho(L) \subseteq \mathrm{GL}(V)$, then $\tau$ is faithful with closed range on $L$.

Proof. If $G=\bigcup_{i=1}^{n} H x_{i}$ is a coset decomposition then $\tau(T)=$ 
$\bigcup_{i=1}^{n} \tau(H) \tau\left(x_{i}\right)$; if $\tau(H)$ is closed, then so is $\tau(G)$. The representation space of $\tau$ is

$$
W=\left\{f: G \longrightarrow V: f(h x)=\rho_{h}[f(x)] \text {, all } x \in G, h \in H\right\} \text {. }
$$

Let $W_{0}=\{f \in W: \operatorname{supp} f \subseteq H\}$ and $W_{1}=\{f \in W: \operatorname{supp} f \cap H=\phi\}$. These are clearly stable under right translation by $H$. If $f \in W$, then $f=\phi \cdot f_{H}+f \cdot \phi_{G H}$ where $\phi_{H}$ is the characteristic function; the summands obviously satisfy the covariance condition defining $W$, so $W=W_{0} \oplus$ $W_{1}$, a direct sum of $H$-modules. Now $W_{0}$ is isomorphic to $V$ under the map $v \mapsto f_{v}$ where $f_{v}$ is the unique element of $W_{0}$ such that $f_{v}(e)=$ $v$. If $h \in H$ and $v \in V$, then $\tau_{h} f_{v}(e)=f_{v}(h)=\rho_{h}(v)=f_{\rho_{h}(v)}(e)$ so that $\tau_{h} f_{v}=f_{\rho_{h}(v)}$ and the map $v \mapsto f_{v}$ is $H$-equivariant. Since $\rho$ is faithful on $L$ and $\rho(L)$ closed, the same is true of $\tau \mid L$ on $W_{0}$, and hence for $\tau \mid L$ on all of $W$ by 3.3 .

Completion of the proof of 3.7. Now $G \times{ }_{\theta} \mathscr{B}$ has finite index in $G \times{ }_{\theta} \mathscr{A}(G)$ since $\mathscr{B}$ has finite index in $\mathscr{A}(G)$. Form the induced representation $J=$ Ind $\left(G \times{ }_{\theta} \mathscr{B} \uparrow G \times{ }_{0} \mathscr{A}(G), \sigma\right)$, a finite dimensional continuous representation of $G \times{ }_{\theta} \mathscr{A}(G)$. By the proof of 3.8, $J \mid G \times{ }_{\theta} \mathscr{B}$ contains a copy of $\sigma$; since $\sigma$ is faithful on $G \times(I), J$ is also. The faithful linear representation $d: \mathscr{A}(G) \rightarrow$ Aut $(\mathfrak{g}) \leqq \mathrm{GL}(\mathrm{g})$, given by the differential, when lifted to $G \times_{\theta} \mathscr{C}(G)$ gives a linear representation $d^{\sim}$ with $\operatorname{ker} d^{\sim}=G \times(I)$. Then $d^{\sim} \oplus J$ is a faithful representation of $G \times{ }_{\theta} \mathscr{A}(G)$. Next we show $\left(d^{\sim} \oplus J\right)(G \times(I))$ is closed; since $J \mid G \times(I)$ is faithful, it suffices, by 3.3, to show that $J(G \times(I))$ is closed. But by 3.8 this follows since $\sigma$ has closed range and is faithful on $G \times(I)$. This proves 3.7.

The following results on faithful representations are not consequences of the results of Hochschild discussed above. We include them since they have proved to be useful in certain situations such as the study of bounded displacement, as in $\S 5$.

THEOREM 3.9. Let $G$ be a faithfully represented connected Lie group with simply connected radical. Let $M$ be any connected Lie group containing $G$ as a normal Lie subgroup, with $M / G$ abelian. Then $M$ has a faithful linear representation.

In particular, if $\alpha \in \mathscr{A}(G)$ lies on a 1-parameter subgroup then $G \times{ }_{\alpha} \boldsymbol{Z}$ is faithfully represented, where $\boldsymbol{Z}$ acts through powers of $\alpha$. If the closed subgroup in $\mathscr{A}(G)$ generated by some power $\alpha^{n}$ of $\alpha$ is compact, then $G \times{ }_{\alpha} Z$ is again faithfully represented, for then $\left\{\alpha^{n k}: k \in Z\right\}_{0}^{-}$is a torus of finite index in $\left\{\alpha^{n k}\right\}^{-}$. Applying 3.9 and then taking induced representations yields this result. 
THEOREM 3.10. Let $G$ be a (not necessarily connected) Lie group with a faithful linear representation $\rho: G \rightarrow \mathrm{GL}(V)$, and let $\alpha \epsilon$ $\mathscr{A}(G)$. If there is a $T \in \mathrm{GL}(V)$ !such that $\rho(\alpha(g))=T \rho(g) T^{-1}$ for all $g \in G$, then $G \times{ }_{\alpha} Z$ has a faithful representation.

In particular, if some power of $\alpha$ is inner, say $\alpha^{p}=\alpha_{g}$, then $\rho\left(\alpha_{g}(x)\right)=\rho(g) \rho(x) \rho(g)^{-1}$ and one again takes an induced representation.

Proof of 3.9. Let $M=R(M) \cdot S$ be the Levi decomposition of and $\pi: M \rightarrow M / G$ the canonical map. Then $\pi(S)$ is a semisimple analytic subgroup of an abelian group and hence is trivial so $S \subseteq G$. It follows that $S$ is a maximal semi-simple analytic subgroup of $G$ and so is a Levi factor of $G$. Since $R(G)$ is characteristic in $G$ and $G$ is normal in $M, R(G)$ is normal in $M$. This means that $R(G)$ is a normal subgroup of $R(M)$. Let $\mathfrak{m}, \mathfrak{g}$, and $\mathfrak{g}$ denote the lie algebras of $M, G$, and $S$ respectively; then we have $\mathfrak{m}=\mathfrak{r}(\mathfrak{m}) \oplus \mathfrak{g}$ and $\mathfrak{g}=\mathfrak{r}(\mathfrak{g}) \oplus$ פ. It follows ithat $\mathfrak{m} / \mathfrak{g} \cong \mathfrak{r}(\mathfrak{m}) / \mathfrak{r}(\mathfrak{g})$. Thus $R(M) / R(G)$ is abelian, so $R(G) \supseteqq[R(M), R(M)]^{-}$. As a simply connected solvable analytic group, $R(G)$ has no nontrivial compact subgroups. The same is true of $[R(M), R(M)]^{-}$. It follows from [15] Theorem 1 that since $S$ has a faithful finite dimensional representation (as a subgroup of $G$ ) and $[R(M), R(M)]^{-}$has no nontrivial compact subgroups, the analytic group $M$ also has a faithful representation.

Proof of 3.10 (adapted from [8]). By induction it follows that $\rho\left(\alpha^{n}(g)\right)=T^{n} \rho(g) T^{-n}$ for all $n \in \boldsymbol{Z}$ and $g \in G$. Define $\varphi: G \times{ }_{\alpha} \boldsymbol{Z} \rightarrow \mathrm{GI}(V)$ by $\varphi(g, n)=\rho(g) T^{n}$ for $g \in G$ and $n \in Z$. Then $\varphi(g, n) \varphi\left(g^{\prime}, n^{\prime}\right)=$ $\rho(g) T^{n} \rho\left(g^{\prime}\right) T^{n^{\prime}}=\rho(g) \rho\left(\alpha^{n}\left(g^{\prime}\right)\right) T^{n} T^{n^{\prime}}=\rho\left(g \alpha^{n}\left(g^{\prime}\right)\right) T^{n+n^{\prime}}=\varphi\left((g, n)\left(g^{\prime}, n^{\prime}\right)\right)$, $\phi$ is a group homomorphism, and if we give $G \times{ }_{\alpha} \boldsymbol{Z}$ the product topology with $\boldsymbol{Z}$ discrete, then $\varphi$ is continuous. Now define $\sigma$ : $G \times{ }_{\alpha} \boldsymbol{Z} \rightarrow \mathrm{GI}(W)$, where $W=V \oplus V$, by

$$
\sigma(g, n)=\left(\begin{array}{c|c}
\varphi(g, n) & 0 \\
\hline 0 & \frac{0}{e^{2 \pi i n n} \varphi(g, n)}
\end{array}\right)
$$

where $g \in G, n \in Z$, and $\lambda$ is an irrational number. Since $e^{2 \pi i \lambda n} I \in$ $Z(\mathrm{GI}(V)), \sigma$ is a continuous representation of $G \times{ }_{\alpha} Z$ on $W$. If $\sigma(g, n)=I$ then $\varphi(g, n)=I=c^{2 \pi i \lambda n} \varphi(g, n)$. Thus $e^{2 \pi i \lambda n}=1$. If $n \neq 0$ then $\lambda$ would be rational, a contradiction. But then $\varphi(g, 0)=\rho(g)=I$. Since $\rho$ is faithful, $g=e$ also.

4. Bounded orbits. In this section we prove 1.1 and 1.3. Here $G$ is a connected Lie group and $\mathscr{B} \cong \mathscr{A}$ subgroups of Aut $(G)$. 
THEOREM 4.1. Let $V$ be a vector group and $\mathscr{A}$ an arbitrary subgroup of $\operatorname{Aut}(V)=\mathrm{GL}(V)$. Then $V_{c}=B(V, \mathscr{A})$ satisfies the conditions (i)-(iii) in 1.3. In the latter part of (iii) we can actually insure that $\operatorname{supp} \mu=(\mathscr{A} x)^{-}$.

Note. At the same time we are, in effect, correcting a gap in Theorem 11 of [7] which is due to a subtle point in applying the result from [16] cited below. That result applies only to closd subgroups of GL $(V)$. As Raghunathan has pointed out to us by way of an example, if $\mathscr{A}$ is not closed then boundedness of all the cyclic subgroups $A^{Z}=\left\{A^{n}: n \in Z\right\}, A \in \mathscr{A}$, does not insure that $\mathscr{A}^{-}$has this property, and so the original group $\mathscr{A}$ need not be relatively compact in GL $(V)$.

Proof. We use the following result on closed linear groups, given as Lemma 7.1 in [16].

LemMA. If $G$ is a closed subgroup of $\mathrm{GL}(V)$ and if all cyclic subgroups $g^{Z}=\left\{g^{n}: n \in Z\right.$ ) are bounded ( $\Leftrightarrow$ compact closure in $\mathrm{GL}(V)$ ) for each $g \in G$, then $G$ is compact.

Since $\mathscr{A} x$ and $\mathscr{A}^{-} x$ have the same closure, it is clear that $B(V, \mathscr{A})=B\left(V, \mathscr{A}^{-}\right)$; moreover, a finite Borel measure on $V$ is $\mathscr{A}$-invariant $\Leftrightarrow$ it is $\mathscr{A}^{-}$-invariant. Thus we may replace $\mathscr{A}$ by $\mathscr{A}^{-}$in proving (i) and (iii). By the following lemma, a layering for $\mathscr{A}^{-}$is automatically a layering for $\mathscr{A}$, so we also make this replacement in dealing with (ii).

Lemma 4.2. Let $G$ be a locally compact group and $G \times X \rightarrow X$ a locally compact $G$-space. For any dense subgroup $H \leqq G$ the bounded elements are the same, $B(X, H)=B(X, G)$. A layering from $X$ down to $Y$ with respect to $G$ is also a layering with respect to $H$.

Proof. Apply the following argument to each layer. If $x \in X_{i} \sim$ $X_{i-1}=L_{i}$ has a relative neighborhood $V$ with $g_{k} V \rightarrow \infty$ in $L_{i}$ for suitable $\left\{g_{k}\right\} \subseteq G$, let $V^{\prime}$ be a neighborhood of $x$ and $U$ a neighborhood of the unit in $G$ such that $U \cdot V^{\prime} \subseteq V$. Choose $h_{k} \in H$ such that $u_{k}=g_{k}^{-1} h_{k} \in U$. For any compact $K \cong L_{i}, h_{k} V^{\prime} \cap K=\phi$ eventually, for if $k_{n(i)}=h_{n(i)} v_{n(i)}^{\prime}=g_{n(i)} u_{n(i)} v_{n(i)}^{\prime}$ with $n(i) \rightarrow \infty$, then $g_{n(i)} V \cap$ $K \neq \phi$, a contradiction.

Thus we may assume $\mathscr{A}$ is closed in GL $(V)$. Since $V$ is a vector space, it is evident that $V_{c}=B(V, \mathscr{C})$ is a vector subspace 
and hence closed, which proves (i). Once (ii) has been proved, we know that any $\mathscr{A}$-invariant, finite Borel measure on $V$ has supp $\mu \subseteq$ $V_{c}$. The rest of (iii) has been done in 2.5. As for (ii), let us pass to the complexification $V_{c}$ and the closed subgroup $\mathscr{A}_{c} \subseteq \operatorname{GL}\left(V_{c}\right)$ corresponding to $\mathscr{A}$. It is clear that $V=V+i 0$ is $\mathscr{K}_{C}$-invariant, that $B(V, \mathscr{A})=(V+i 0) \cap B\left(V_{c}, \mathscr{C}_{c}\right)$, and that a layering in $V_{c}$ induces a layering in $V$ by intersection with $V+i 0$. Therefore we may assume $V$ is a vector space over $C$.

Each $T \in \mathscr{A}$ has a Jordan decomposition $T=T_{s} \cdot T_{u}$ (commuting operators, not necessarily back in $\mathscr{A}$ ) such that $T_{u}$ is unipotent and $T_{s}$ diagonalizable. For $T \in \mathscr{A}$ let $V_{T}=\boldsymbol{C}$-span $\left\{v \in V: T_{u} v=v, T_{s} v=\right.$ " $v$ for some $|\mu|=1\}$; this vector space consists precisely of the points $v$ with bounded orbit under the cyclic subgroup $T^{\prime \prime}$ generated by $T$. [If $W$ is the set of $v$ with bounded $T^{Z}$-orbit, this is a $T^{Z_{\text {- }}}$ invariant subspace, so $T^{z}$ induces a bounded group of linear operators on $W$. For a suitable inner product on $W, T^{Z} \mid W$ consists of unitary operators, so that $T \mid W$ is diagonalizable, $T_{u} w=w$ on $W$, and $W \subseteq$ $V_{T}$. The converse inclusion is obvious.] Let $V_{*}=\cap\left\{V_{T}: T \in \mathscr{\mathscr { A }}\right\}$, the subspace of vectors bounded under the iterated action of each operator $T \in \mathscr{A}$. Obviously this $\mathscr{A}$-invariant subspace contains $V_{c}$.

We assert that there is an $\mathscr{A}$-invariant layering from $V$ down

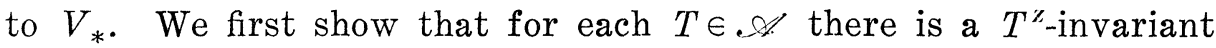
layering from $V$ down to $V_{T}$. Then we invoke some straightforward general facts about layerings proved in [5]; a slight rewording of the discussion there shows they are valid for the strengthened notion of layering used in this paper. By [5], Lemma 8.6, we can replace the $T^{\prime \prime}$-invariant layering down to $V_{T}$ with an $\mathscr{A}$-invariant layering down to some $\mathscr{A}$-invariant subspace $U_{T}$ such that $V_{T} \supseteqq$ $U_{T} \supseteq V_{*}$. (Note: The connectedness hypothesis mentioned in that lemma is superfluous, so it may legitimately be applied here.) Then by [5], Lemma 2.2, there will be an $\mathscr{A}$-invariant layering from $V$ down to $V_{*}$. Notice that finitely many $V_{T}$ intersect to give $V_{*}$.

To produce the $T^{\prime \prime}$-invariant layering from $V$ down to $V_{T}$, it is not hard to adapt the self-contained discussion of Proposition 8.1 of [5] to actions of $\boldsymbol{Z} \times V \rightarrow V$ instead of $\boldsymbol{R} \times V \rightarrow V$. Actually, the arguments in [5] can be simplified, so for the sake of completeness we give these details. [If $T \in \mathscr{A}$ and $V^{\prime}$ is a $T$-invariant subspace then $V_{T}^{\prime}=V^{\prime} \cap V_{T}$; furthermore, the Jordan decomposition of $T \mid V^{\prime}$ is $\left(T_{s} \mid V^{\prime}\right)\left(T_{u} \mid V^{\prime}\right)$. By induction on dimension of $V$ it suffices to show that if $V \neq V_{T}$, there is a $T$-invariant subspace $V \neq V^{\prime} \supseteqq V_{T}$ such that $V \sim V^{\prime}$ satisfies the layer condition. A unipotent operator such as $T_{u}$ lies on a 1-parameter subgroup and has the form $T_{u}=$ $e^{N}$ for some nilpotent $N$, hence $\left\|T_{u}^{n}\right\| \leqq p(n)=\sum_{k=0}^{s}\left|n^{k}\right|\|N\|^{k} / k !$; then, $\|v\| / p(n) \leqq\left\|T^{n} v\right\| \leqq p(n)\|v\|$ for all $v \in V, n \in \boldsymbol{Z}$. Let $V=$ 
$\bigoplus_{i=1}^{r} V_{i}$ be the decomposition of $V$ into the distinct eigenspaces of $T_{s}$, say with $T_{s} v_{j}=\mu_{j} v_{j}$ for $v_{j} \in V_{j}$. Let $V^{\prime}=\bigoplus\left\{V_{j}:\left|\mu_{j}\right|=1\right\}$. If $v=v_{1}+\cdots+v_{r} \notin V^{\prime}$ then $v_{j} \neq 0$ for some index $j$ such that $\left|\mu_{j}\right| \neq$ 1. Take $\delta=\left\|v_{j}\right\|$, and $K>\delta>0$, and form the neighborhood $W=$ $\left\{w \in V:\left\|w_{i}\right\| \leqq K\right.$ all $\left.i,\left\|w_{j}\right\| \geqq \delta / 2\right\}$, where $\|v\|$ is any convenient norm such that $\|v\|^{2}=\left\|v_{1}\right\|^{2}+\cdots+\left\|v_{r}\right\|^{2}$. Then for all $n \in Z, w \in$ $W$,

$$
\left\|T^{n} w\right\|^{2}=\left\|T_{u}^{n}\left(\sum \mu_{i}^{n} w_{i}\right)\right\|^{2} \geqq\left|\mu_{j}\right|^{2 n}\left\|T_{u}^{n} w_{j}\right\|^{2} \geqq\left|\mu_{j}\right|^{2 n} \delta^{2} / 4 p(n)^{2} .
$$

Letting $n \rightarrow \infty$, we can insure that $\inf \left\{\left\|T^{n} w\right\|: w \in W\right\} \rightarrow+\infty$, as required.

Thus we may assume $\left|\mu_{i}\right|=1$, all $i: T_{s}$ generates a bounded (unitarizable) group of operators on $V$. If $T_{u}=I$, then $V=V_{T}$ and there is nothing to prove. If $T_{u}=e^{N} \neq I$, then $e^{t_{N}}$ commutes with $T_{s}$ for all $t \in Z$, hence for all $t \in \boldsymbol{R}$ since $e^{t N}$ is a polynomial. Thus $T_{s}$ commutes with the infinitesimal generator $N$, and the kernels $\quad(0) \varsubsetneqq \operatorname{Ker} N \varsubsetneqq \cdots \varsubsetneqq \operatorname{Ker} N^{m-1} \varsubsetneqq \operatorname{Ker} N^{m}=V \quad(m \geqq 2$ since $N \neq 0$ ) are invariant under $T_{s}, T$. Let $V^{\prime}=\operatorname{Ker} N^{m-1}, V^{\prime \prime}=\operatorname{Ker}$ $N^{m-2}$. In demonstrating the layering property for a point $v_{0} \in V \sim$ $V^{\prime}$ we may assume $V^{\prime \prime}=(0)$; if not, we can pass to the induced operators $N^{\sim}, T^{\sim}$, and $T_{u}^{\sim}=e^{N^{\sim}}$ on $V / V^{\prime \prime}$ and lift our conclusions back to $V$. If $V^{\prime \prime}=(0)$, then $v_{0} \in V \sim V^{\prime} \Rightarrow N\left(v_{0}\right) \in V^{\prime} \sim V^{\prime \prime}$, so $N v_{0} \neq 0$, while for any $v \in V$ we have $T_{u}^{k}(v)=e^{k N} v=v+k N(v)$. Pick any open neighborhood $W$ of $N v_{0}$ that is bounded away from zero and let $n(1)<n(2)<\cdots$ be chosen so that $n(k) W \rightarrow \infty$ in $V$. Then take any compact neighborhood $U \subseteq V \sim V^{\prime}$ of $v_{0}$ such that $N(U) \subseteq$ $W$. We get

$$
\begin{aligned}
& T_{u}^{n(k)}(U)=e^{n(k) N}(U) \subseteq U+n(k) N(U)=n(k)\left[\frac{1}{n(k)} U+N(U)\right] \\
& T_{u}^{n(k)}(U) \leqq n(k) W \cap\left(V \sim V^{\prime}\right)
\end{aligned}
$$

for all large $k$. Obviously then $T_{u}^{n(k)}(U) \rightarrow \infty$ in $V \sim V^{\prime}$.]

Thus we get an $\mathscr{A}$-invariant layering from $V$ down to $V_{*}$. Now, each cyclic subgroup in $\mathscr{A} \mid V_{*}$ is bounded in GL $\left(V_{*}\right)$ since $T \in \mathscr{A} \Rightarrow T\left|V_{*}=T_{s}\right| V_{*} ;$ if $\mathscr{A} \mid V_{*}$ were a closed subgroup of GL $\left(V_{*}\right)$, we could invoke the lemma from [16] mentioned above to conclude that $\mathscr{A} \mid V_{*}$ is compact, from which it would follow that $V_{*}=V_{c}$. However (and this is the gap in [7]), there seems to be no reason $\mathscr{A} \mid V_{*}$ should be closed, and we know that boundedness of the cyclic subgroups is not a property which can be passed from $\mathscr{A} \mid V_{*}$ to its closure in GL $\left(V_{*}\right)$. We circumvent these difficulties as follows. Write $V_{1}=V_{*}, \mathscr{A}_{1}=\mathscr{A} \mid V_{1}$ with closure $\mathscr{X}_{1}^{-} \subseteq \mathrm{GL}\left(V_{1}\right)$. Since a group and its closure determine the same subspace of bounded 
vectors, we have

$$
V_{c}=B(V, \mathscr{A})=B\left(V_{1}, \mathscr{A}_{1}\right)=B\left(V_{1}, \mathscr{C}_{1}^{-}\right) \subseteq V_{*} .
$$

As above, there is a layering from $V$ to $V_{1}=V_{*}$ under the action of $\mathscr{A}$. In passing from $\mathscr{A}_{1}$ (all $T \in \mathscr{A}_{1}$ are semisimple with eigenvalues of absolute value 1 ) to $\mathscr{A}_{1}^{-}$we may pick up operators having unipotent part, or eigenvalues $|\mu| \neq 1$; thus $\left(V_{1}\right)_{*}=\cap\left\{\left(V_{1}\right)_{T}: T \in\right.$ $\left.\mathscr{A}_{1}^{-}\right\}$may be smaller that $V_{1}$. If so, we begin a finite induction. Define $V_{2}=\left(V_{1}\right)_{*}, \mathscr{A}_{2}=\mathscr{L}_{1}\left|V_{2}=\mathscr{A}\right| V_{2}$ with closure $\mathscr{A}_{2}^{-} \subseteq \operatorname{GL}\left(V_{2}\right)$. Again, there is a layering from $V_{1}$ down to $V_{2}$ under the action of $\mathscr{A}_{2}{ }^{-}$, but this is in fact a layering with respect to the action of $\mathscr{A}\left(\operatorname{alias} \mathscr{A}_{2}\right)$ in view of 4.2. Thus we get a layering from $V$ (through $\left.V_{1}\right)$ to $V_{2}$ under the action of $\mathscr{A}$. If, once again, $\left(V_{2}\right)_{*}=$ $\cap\left\{\left(V_{2}\right)_{T}: T \in \mathscr{A}_{2}^{-}\right\} \neq V_{2}$ we continue, defining $V_{3}=\left(V_{2}\right)_{*}, \mathscr{A}_{3}=\mathscr{A}_{2} \mid V_{3}=$ $A \mid V_{3}$ with closure $\mathscr{L}_{3}$ in $\mathrm{GL}\left(V_{3}\right)$, and so on. This process must stop, say at the $k$ th step since we lose dimension. We now arrive at a layering from $V$ (through $V_{1}, \cdots, V_{k-1}$ ) down to $V_{k}$ and we have $V_{k}=\left(V_{k}\right)_{*}=\cap\left\{\left(V_{k}\right)_{T}: T \in \cdot \mathscr{A}_{k}{ }^{-}\right\}$. Thus each $T \in \mathscr{O}_{k}^{-}$is semisimple with eigenvalues of modulus one. Since $\mathscr{A}_{k}^{-}$is a closed linear group we now apply [16], Lemma 7.1, to conclude that $\mathscr{A}_{k}^{-}$is compact. Thus $\left(V_{k}\right)_{*}=B\left(V_{k}, \mathscr{A}_{k}^{-}\right)=V_{c}$. This completes the proof of (ii), and hence of 3.1 .

It is easy to extend this to continuous affine actions on $V$ by imbedding $V$ as a hyperplane in a larger space $W$, and realizing the action as the restriction of a linear action on the larger space, see the discussion of 2.7. We now make a crucial generalization of this simple idea.

Proof of 1.3. In $G$ let $K$ be the largest compact connected subgroup in the nilradical $N$. This is actually a characteristic subgroup of $G$ and $G / K$ has simply connected nilradical $N / K$. [By examining the adjoint action of $K$ on $\mathfrak{n}$, we see that any compact connected subgroup is central in $N$, hence $K$ is just the largest compact connected subgroup normal in $N$. The latter description is canonical, so $K$ is characteristic in $G$ - invariant under Aut $(G) . N / K$ is clearly simply connected, having no proper compact subgroups. Next let $N^{\prime}$ be the inverse image under $\pi: G \rightarrow G / K$ of $N(G / K) ; N^{\prime}$ is normal and eventually we have $\left[\mathfrak{n}^{\prime},\left[\cdots\left[\mathfrak{n}^{\prime}, \mathfrak{n}^{\prime}\right] \cdots\right] \subseteq \mathfrak{f}\right.$. Suppose $X \in \mathfrak{n}^{\prime}$. Then the connected group of conjugations $\alpha_{\exp t x} \mid K$ lies in the identity component (Aut $K)_{0}$, which by a result of Iwasawa [11], Theorem 1 , is equal to $\operatorname{Int}(K)$. Since $K$ is abelian, we get $[X, \mathfrak{k}]=$ 0 and $\left[\mathfrak{n}^{\prime}, \mathfrak{k}\right]=0$, so $\mathfrak{f}^{\prime}$ is a nilpotent ideal in $\mathfrak{g}$. Hence $\mathfrak{n t}^{\prime}=\mathfrak{t}$ and 
$N(G / K)=N / K]$. Let $\mathscr{C}^{\prime}$ be the induced subgroup of Aut $(G / K)$ and suppose 1.3 proven for $G / K$ and $\mathscr{A}^{\prime}$. Since $K$ is compact it is clear that; (i) $\mathscr{A} x$ is bounded $\Leftrightarrow \mathscr{A}^{\prime} \pi(x)$ is bounded in $G / K$, so that $B(G, \mathscr{A})=\pi^{-1}\left(B\left(G / K, \mathscr{A}^{\prime}\right)\right)$ is closed, and (ii) an $\mathscr{A}^{\prime}$-invariant layering in $G / K$ pulls back to an $\mathscr{A}$-invariant layering in $G$. Now (i)+ (ii) $\Rightarrow$ finite $\mathscr{A}$-invariant Borel measures must be supported within $B(G, \mathscr{A})$. Conversely, if $x \in B(G, \mathscr{A})$ then $\pi x \in B\left(G / K, \mathscr{A}^{\prime}\right)$ and there is an $\mathscr{A}^{\prime}$-invariant measure $\mu^{\prime}$ such that $\pi x \in \operatorname{supp} \mu^{\prime} \leqq B(G / K$, $\left.\mathscr{A}^{\prime}\right)$. This lifts back to an $\mathscr{A}$-invariant measure on $G$ if we take

$$
\langle\mu, f\rangle=\left\langle\mu^{\prime}, \int_{K} f(x k) d k\right\rangle \text { all } f \in C_{c}(G) \text {. }
$$

Obviously $x \in \operatorname{supp} \mu=\pi^{-1}\left(\operatorname{supp} \mu^{\prime}\right) \subseteq B(G, \mathscr{A})$.

Thus we may assume that $G$ has simply connected nilradical. (The faithful representability of Levi factors is unaffected by this transition.) By 3.7 there is a faithful linear representation $\theta: G \times{ }_{\sigma}$ Aut $(G) \rightarrow \mathrm{GL}(V)$ such that $G^{\prime}=\theta(G \times I)$ is a closed subgroup of $\mathrm{GL}(V)$. By imbedding GL $(V)$ as SL $(V \oplus \boldsymbol{R})$ in the usual way, we can assume that range $(\theta) \subseteq \mathrm{SL}(V)$, so that $G^{\prime}$ is a closed set in the space $W=$ End $(V)$ of all linear operators, and $\theta: G \rightarrow G^{\prime}$ is a topological isomorphism. Let $\mathscr{A}^{\prime}=\theta(e \times \mathscr{A})$. Now the action of $\mathscr{A}$ on $G$ is realized as the action of $\mathscr{A}^{\prime}$ by similarity transformations on the closed $\mathscr{C}^{\prime}$-invariant set $G^{\prime} \subseteq$ End $(V)$ : if $A=\theta(e, \alpha)$ and $X=$ $\theta(g, I)$, we have

$$
\theta(\alpha(g))=\theta\left((e, \alpha) \cdot(g, I) \cdot(e, \alpha)^{-1}\right)=A X A^{-1} \text { all } g \in G, \alpha \in \mathscr{A} .
$$

The action $\mathscr{A}^{\prime} \times W \rightarrow W$ via $(A, X) \rightarrow A X A^{-1}$ is a linear action on $W$. Since $G^{\prime}$ is closed, $\mathscr{A}^{\prime}$-invariant (and closedness of $G^{\prime}$ is absolutely essential here!), we have

$$
B\left(G^{\prime}, \mathscr{A}^{\prime}\right)=B\left(W, \mathscr{A}^{\prime}\right) \cap G^{\prime},
$$

and any layering from $W$ down to $B\left(W, \mathscr{A}^{\prime}\right)$ by closed $\mathscr{A}^{\prime}$-invariant sets obviously induces such a layering from $G^{\prime}$ down to $B\left(G^{\prime}, \mathscr{A}^{\prime}\right)$. Since $\theta: G \rightarrow G^{\prime}$ is equivariant with respect to $\mathscr{A}, \mathscr{A}^{\prime}, B(G, \mathscr{A})$ identifies with $B\left(G^{\prime}, \mathscr{A}^{\prime}\right)$. Thus (i) and (ii) follow for $G$, from our previous result 4.1 on linear actions; as usual, (i) + (ii) $\Rightarrow$ any finite $\mathscr{A}$-invariant Borel measure has support in $B(G, \mathscr{A})$. As for the converse, let $g \in B(G, \mathscr{A}), X=\theta(g) \in G^{\prime}$. For the linear action of $\mathscr{A}^{\prime}$ we can actually find an $\mathscr{A}^{\prime}$-invariant measure $\mu$ on $W$ such that $\operatorname{supp} \mu=\left(\mathscr{A}^{\prime} \cdot X\right)^{-}$, see 4.1 . But $\left(\mathscr{A}^{\prime} \cdot X\right)^{-} \subseteq G^{\prime}$, so $\mu$ is really a nonzero measure on $G^{\prime}$ with $X \in \operatorname{supp} \mu \subseteq B\left(G^{\prime}, \mathscr{A}^{\prime}\right)$. This can be lifted back to $G$ as required to finish the proof of part (iii). This completes the proof of 1.3 . 
Note. In the nonlinear case, unless the nilradical is simply connected we do not seem to be able to take $x \in B(G, \mathscr{A})$ and find an $\mathscr{A}$-invariant measure $\mu$ with $\operatorname{supp} \mu=(\mathscr{A} \cdot x)^{-}$, rather than $(\mathscr{A} \cdot x)^{-} \subseteq$ supp " @ $\cong(G, \mathscr{A})$. The possibility of doing this gets lost during the transition from $G$ to $G / K$ if we have to deal with compact subgroups in the nilradical.

The density Theorem 1.1 now follows easily from 1.3.

Proof of 1.1. We may assume $\mathscr{A}, \mathscr{B}$ are closed in Aut $(G)$, see 2.10. Let $x \in G$ have bounded $\mathscr{B}$-orbit. Then there is an $\mathscr{B}$-invariant finite Borel measure $\mu \geqq 0$ such that $x \in \operatorname{supp} \mu \subseteq B(G, \mathscr{B})$. Pushing this around by elements of $\mathscr{A}$ and taking an average over $\mathscr{A} / \mathscr{B}$ we get a finite $\mathscr{A}$-invariant measure $\nu$ on $G$ : define $\left\langle T_{\alpha} \mu\right.$, $f\rangle=\langle\mu, f \circ \alpha\rangle$ for $\alpha \in \operatorname{Aut}(G)$, so that $T_{\alpha \beta} \mu=T_{\alpha} \mu$ for all $\alpha \in \mathscr{A}$, $\beta \in \mathscr{B}$. If $\lambda$ is the finite volume on $\mathscr{A} / \mathscr{B}$ we take

$$
\langle\nu, f\rangle=\int_{, .}\left\langle T_{\alpha} \mu, f\right\rangle d \lambda(\alpha) \text { all } f \in C_{c}(G) \text {. }
$$

Clearly $\|\nu\|<\infty, T_{\alpha} \nu=\nu$ for all $\alpha \in \mathscr{A}$, and

$$
\operatorname{supp} \nu=\left(U \operatorname{supp} T_{\alpha} \mu\right)^{-} \text {. }
$$

Thus we get $x \in \operatorname{supp} \mu \subseteq \operatorname{supp} \nu \subseteq B(G, \mathscr{A})$ and $\mathscr{A} x$ is bounded as required.

Can one drop the condition on the Levi factor in $G$, thereby obtaining 1.1-1.3 for all connected $G$ ? There is a natural test case: $G$ a simply connected semisimple Lie group with infinite center. By direct calculations, which we omit, we have shown that the results are true when $G$ is the covering group of SL $(2, R)$. For arbitrary simply connected semisimple groups, it would suffice to verify that closures of conjugacy classes have the following geometric property: let $\pi: G \rightarrow G / Z(G)=G_{1}$ be the covering homomorphism. For $x \in G$, $y \in G_{1}$ let $C_{x}, C_{y}$ be their conjugacy classes and let $\bar{C}_{x}, \bar{C}_{y}$ be their closures.

Conjecture. If $x \in G$ and $y=\pi(x)$ then $\pi: \bar{C}_{x} \rightarrow \bar{C}_{y}$ is surjective and proper (inverse image of compact is compact).

If $x$ is a semisimple element, then $C_{x}$ is already closed and the desired property follows easily; however, to derive 1.3 one needs to know that all classes have the property. Further work on this problem will be reported later.

Remark on bounded orbits. If $G \times X \rightarrow X$ is a locally compact $G$-space, an element $x$ with bounded orbit $G \cdot x$ may actually have 
the property:

There is a neighborhood $U$ of $x$ with infinitely many disjoint transforms $\left\{g_{j} \cdot U: j=1,2, \cdots\right\}$.

For the actions of automorphisms we have been considering, $\mathscr{A} \times$ $G \rightarrow G$ where $\mathscr{A} \subseteq \operatorname{Aut}(G)$, this kind of behavior cannot occur; hence, the terminal set in any $\mathscr{A}$-invariant layering $G=X_{0} \supseteqq \cdots \supseteqq$ $X_{m}$ must contain $B(G, \mathscr{A})$. There does not seem to be direct proof of this from general arguments about compactness, etc. However, it does follow from part (iii) of 1.3: if $x \in B(G, \mathscr{A})$ then there is a finite, $\mathscr{A}$-invariant Borel measure with $x \in \operatorname{supp} \mu$. Since $\|\mu\|<\infty$, $\operatorname{supp} \mu$ is forced to lie within the terminal set for any layering, so $B(G, \mathscr{A}) \subseteq X_{m}$; in particular, property $\left(^{*}\right)$ cannot hold for $x$.

In [5] there is a (harmless) misstatement concerning this point, see pp. $226(5 \mathrm{~b})$ and $227(5 \mathrm{t})$. These remarks are never really used in the paper and may be deleted. Actually, the assertion being made is true, as a consequence of the main theorems of the paper; it does not seem to follow from general compactness arguments, as implied in the original lines.

5. Bounded displacement of automorphisms. We now take up discussion of Theorem 1.2, and some of its variants.

Lemma 5.1. Let $G$ be a connected Lie group, $A \supseteq B$ closed subgroups, and $\alpha \in \operatorname{Aut}(G)$. For any compact characteristic subgroup $K \subseteq G$, form $G^{\sim}=G / K, A^{\sim}=A K / K, B^{\sim}=B K / K$, and let $\tilde{\alpha} \in \operatorname{Aut}\left(G^{\sim}\right)$ be the induced automorphism. Then

(i) $A / B$ has finite volume $\Leftrightarrow A^{\sim} / B^{\sim}$ does.

(ii) $\operatorname{disp}(\alpha, A)$ is bounded $\Leftrightarrow \operatorname{disp}\left(\tilde{\alpha}, A^{\sim}\right)$ is bounded.

If $K$ contains the largest compact connected subgroup in the nilradical $N$, then $N(G / K)=N K$ and $N(G / K)$ is simply connected.

Proof. Clearly $A^{\sim}, B^{\sim}$ are closed in $G^{\sim}$. In (i), $\Leftrightarrow$ follows from 2.10 and $(\Longleftrightarrow)$ from compactness of $K$. In (ii), $\Leftrightarrow)$ is trivial and the converse follows since $\pi^{-1}\left(\operatorname{disp}\left(\widetilde{\alpha}, A^{\sim}\right)\right) \supseteqq \operatorname{disp}(\alpha, A)$ is compact. The last point follows from the remarks at the beginning of the proof of 1.3 .

Now we are ready to prove 1.2.

THEOREM 5.2. Let $G$ be any connected Lie group with Levi factor $S$ which is faithfully represented. Let $B \cong A$ be closed subgroups of $G$ such that $A / B$ has finite volume, and let $\alpha \in \operatorname{Aut}(G)$. If $\operatorname{disp}(\alpha, B)$ is bounded then $\operatorname{disp}(\alpha, A)$ is bounded. 
Proof. Factoring out the largest compact connected subgroup in $N$ (=largest compact connected subgroup normal in $N$ ), we may assume that $N$ is simply connected in view of 5.1 ; this does not affect the condition on the Levi factor. In $G \times{ }_{\sigma}$ Aut $(G)$ we have $(g, I)(e, \alpha)(g, I)^{-1}=\left(g \alpha(g)^{-1}, \alpha\right)$ for all $g \in G$. If we now consider $g$ lying in either $A$ or $B$, it follows that the orbit $O_{B}(e, \alpha)$ under conjugation by $B \times I$ is bounded $\Leftrightarrow \operatorname{disp}(\alpha, B)$ is bounded, and similarly for the orbit $O_{A}(e, \alpha)$ under $A \times I$. Now take a faithful representation $\theta: G \times{ }_{\sigma} \operatorname{Aut}(G) \rightarrow \mathrm{GL}(V)$ as in 3.7, such that $G^{\prime}=\theta(G \times I)$ is closed. By making a further imbedding of GL $(V)$ into $\mathrm{SL}(V \oplus \boldsymbol{R})$ we may assume $G^{\prime}$ is a closed set in $W=$ End $(V)$. Now $G \times(\alpha)$ is invariant under congujation by elements of $G \times I$, and $S=\theta(G \times(\alpha))$ is a closed $G^{\prime}$-invariant set in $W$ if we let $G^{\prime}$ act on $W$ by similarity transforms, $X \rightarrow A X A^{-1}\left(A \in G^{\prime}, X \in\right.$ End $\left.(V)\right)$. Obviously $\theta: G \times(\alpha) \rightarrow$ $S$ is an equivariant homeomorphism. Letting $A^{\prime}=\theta(A \times I), B^{\prime}=$ $\theta(B \times I)$, it follows from 1.1 that these have the same bounded elements in $W: B\left(W, A^{\prime}\right)=B\left(W, B^{\prime}\right)$. Since $S$ is closed, $B\left(S, A^{\prime}\right)=$ $S \cap B\left(W, A^{\prime}\right)=S \cap B\left(W, B^{\prime}\right)=B\left(S, B^{\prime}\right)$. Transferring this back to $G \times(\alpha)$, we see that $O_{B}(e, \alpha)$ bounded $\Rightarrow O_{A}(e, \alpha)$ bounded, which proves the theorem.

The restriction on the Levi factor in 5.2 can be removed in certain special circumstances. We have not, as yet, been able to prove Theorems 1.1 and 1.3 in these cases.

Proposition 5.3. Let $G$ be a connected Lie group, $H$ a closed subgroup with $G / H$ of finite volume, and $\alpha \in$ Aut $(G)$. Take the linear Lie group $\operatorname{Ad}(G)$ (in its Lie topology $\cong G / Z(G)$ ) and suppose that $\mathrm{Ad}(G)$ has no automorphisms of bounded displacement. If $\operatorname{disp}(\alpha, H)$ is bounded, then $\alpha$ has bounded displacement on all of $G$.

Proof. Clearly $\alpha$ induces an $\tilde{\alpha} \in \operatorname{Aut}(\operatorname{Ad}(G))$. If $\pi: G \rightarrow \operatorname{Ad}(G)$ is the adjoint representation, then $\mathrm{Ad}(G) / \pi(H)^{-}$has finite volume by 2.10 . Since $\operatorname{disp}(\widetilde{\alpha}, \pi(H))$ is bounded, the same is true of disp $\left(\widetilde{\alpha}, \pi(H)^{-}\right)$. Since $\operatorname{Ad}(G)$ is a linear Lie group, its Levi factor is faithfully represented; thus, by $5.2, \operatorname{disp}(\tilde{\alpha}, \operatorname{Ad}(G))$ is bounded, hence $\widetilde{\alpha}=i d$. This means $\dot{\phi}(g)=\alpha(g) g^{-1} \in Z(G)$. It follows that the function $\phi: G \rightarrow Z(G)$ is a continuous homomorphism. In particular $\phi(H)^{-}$ and $\dot{\phi}(G)^{-}$are closed subgroups of $Z(G)$ with $\phi(H)^{-}$compact, since $\operatorname{disp}(\alpha, H)$ is bounded. By $2.10, \dot{\phi}(G)^{-} / \dot{\phi}(H)^{-}$has finite volume, and is hence compact since $\phi(G)^{-}$is abelian. Thus $\phi(G)^{-}$is compact and $\alpha$ has bounded displacement.

As noted in the proof, disp $(\alpha)$ in fact lies in a compact central 
subgroup of $G$.

CoRollary 5.4. Let $G$ be either minimally almost periodic, complex analytic, or reductive. If $H$ is a closed subgroup with $G / H$ of finite volume and if $\alpha \in \operatorname{Aut}(G)$, then $\operatorname{disp}(\alpha, H)$ is bounded $\Leftrightarrow \operatorname{disp}(\alpha, G)$ is bounded.

Proof. If $G$ is complex analytic, then the Levi factor is faithfully represented and this result already follows from 5.2. If $G$ is minimally almost periodic, so is $\mathrm{Ad}(G)$, which therefore has no automorphisms of bounded displacement [14]; thus we may apply 5.3.

If $G$ is reductive then $G=Z(G)_{0} \cdot[G, G]$ where $[G, G]$ is semisimple. The largest compact normal connected subgroup $K(G)$ contains the product $C$ of the compact factors in $[G, G]$, since $C$ is normal; by 5.1 we may assume that $C$ is trivial. But then $\operatorname{Ad}(G)=$ $\operatorname{Ad}([G, G])$ also has no compact factors, and therefore has no automorphisms of bounded displacement [18].

CoRollaRY 5.5. Let $G$ be a connected Lie group, $H$ a closed subgroup with $G / H$ of finite volume, and let $\alpha \in$ Aut $(G)$. Suppose that $G / R$ has no compact factors, and that the radical $R$ has a type $E$ Lie algebra or is 2-step solvable. If $\operatorname{disp}(\alpha, H)$ is bounded, then $\alpha$ has bounded displacement.

Proof. If $R$ is not simply connected then it has a proper central torus [14], which by 5.1 we may assume is absent. Assuming $R$ simply connected, the proof works by induction on the degree $d$ of solvability of $R$. If $d=0$ then $G$ is semisimple without compact factors and so is minimally almost periodic, and the result follows from 5.4. In general, let $V$ be the last proper term in the derived series for $R$, a closed vector group characteristic in both $R$ and $G$. Then $R / V$ is simply connected solvable of type $E$ and the radical of $G / V$ has lower degree of solvability; also, $G / R \cong(G / V) /(R / V)$ and there are no compact factors. Now $\alpha$ induces $\tilde{\alpha} \in \operatorname{Aut}(G / V)$ and $\operatorname{disp}\left(\tilde{\alpha}, \pi(H)^{-}\right)$is bounded, where $\pi: G \rightarrow G / V$ is the canonical map. By induction, $\tilde{\alpha}$ has bounded displacement on $G / V$; but by [14] it must be trivial on $G / V$, so $\phi(g)=\alpha(g) g^{-1} \in V$ for all $g \in G$. Now $\phi: G \rightarrow V$ is a continuous map which satisfies the cocycle identity: $\phi\left(g_{1} g_{2}\right)=\phi\left(g_{1}\right) \cdot g_{1} \phi\left(g_{2}\right) g_{1}^{-1}$. If $\mathfrak{v}$ denotes the Lie algebra of $V$ then exp: $\mathfrak{b} \rightarrow V$ is a diffeomorphism, and $\psi(g)=\log \phi(g)$ is bounded on a subset of $G$ if and only if $\phi$ is. By the Campbell-Hausdorff formula, since $V$ is abelian and $\operatorname{Int}(G)$-stable, we get

$$
\begin{aligned}
\psi\left(g_{1} g_{2}\right) & =\log \left(\phi\left(g_{1}\right) \cdot g_{1} \phi\left(g_{2}\right) g_{1}^{-1}\right)=\log \phi\left(g_{1}\right)+\log \left(g_{1} \phi\left(g_{2}\right) g_{1}^{-1}\right) \\
& =\psi\left(g_{1}\right)+\operatorname{Ad}\left(g_{1}\right)\left(\psi\left(g_{2}\right)\right) .
\end{aligned}
$$


Thus $i$ is a 1-cocycle with respect to the adjoint representation of $G$ on $\mathfrak{v}$. Since $\operatorname{disp}(\alpha, H)$ is bounded, $\phi \mid H$ is bounded and so is $\psi \mid H$. By $2.6 \psi$ is bounded on $G$, and so is $\phi ; \alpha$ has bounded displacement.

If $R$ is 2 -step solvable then $[R, R]^{-}$is abelian, and is the direct product $V \times T$ of a vector group and a torus. The $T$ is characteristic in $G$, and by 5.1 may be assumed to be trivial. Now $G / V$ has an abelian (type $E$ ) radical. By the previous paragraph, $\widetilde{\alpha} \in$ Aut $(G / V)$ is trivial, so $\phi(g)=\alpha(g) g^{-1} \in V$ for all $g \in G$. Now we may again argue as above.

6. Infinite dimensional representations. In this section we deal with two infinite dimensional analogs of the $H$-fixed/G-fixed results of [14]. One of these uses a recent generalization of the Borel density theorem, given in [13]. If $G$ operates on $X$ then it also operates on $M_{c}(X)=$ finite Borel measures with compact support, giving rise to an infinite dimensional linear representation.

THEOREM 6.1. Let $G$ be a locally compact group, $H$ a closed subgroup with $G / H$ of finite volume, and $G \times V \rightarrow V$ a linear action on a finite dimensional vector space $V$. Assume that

(i) $G$ is minimally almost periodic, or

(ii) $G$ is complex analytic with holomorphic action, or

(iii) $G$ is solvable linear Lie group with only real eigenvalues. Then any $\mu \in M_{c}(V)$ which is $H$-invariant is also G-invariant. Furthermore, if

(iv) $G$ is a linear Lie group such that $G / R$ has no compact factors, and the radical $R$ satisfies (ii),

then the same conclusion holds.

Proof. If $\mu$ is $H$-invariant this means it is $H$-fixed under the action of $G$ on $M_{c}(V)$. By 2.3, it is $H^{\sharp}$-fixed. But by [13] (or in case (i), by [2]) these conditions imply that $\mu$ is $G$-fixed; therefore $f$ is a $G$-invariant measure.

We now turn to quite general, but unitary, representations on a Hilbert space $V$. $\mathscr{C} \mathscr{S}(V)$ denotes the Hilbert-Schmidt operators on $V$. The following is a well known result of Mackey [12].

THEOREM 6.2. Let $\rho$ be a strongly continuous unitary representation of the locally compact group $G$ on a Hilbert space $V$. Let $G$ act on $\mathscr{K} \mathscr{S}(V)$ by $(g, T) \rightarrow \rho_{g} T \rho_{g}^{-1}$. Then this representation is equivalent to $\rho \otimes \bar{\rho}$ on $V \otimes \bar{V}$. Hence it is a strongly continuous representation of $G$ on $\mathscr{C} . \mathscr{P}(V)$. It is weakly holomorphic if $G$ is 
complex analytic and $\rho$ is weakly holomorphic.

The following extends a result of S. P. Wang [21], see also [20]; 6.3 below is also an analog of results in [13]. Here the representations are unitary and infinite dimensional, while those of [13] were nonunitary and finite dimensional.

THEOREM 6.3. Let $G$ be a locally compact group, $H$ a closed subgroup with $G / H$ of finite volume, and $\rho$ a strongly continuous unitary representation of $G$ on the Hilbert space $V$. Then the $H$ fixed and $G$-fixed vectors coincide in $\mathscr{H} \mathscr{S}(V)$ :

$$
Z_{\mathscr{P S}(V)}(G)=Z_{\mathscr{C S}(V)}(H) \text {. }
$$

Moreover, if

(i) $G$ is minimally almost periodic, or

(ii) $G$ is complex analytic and $\rho$ weakly holomorphic, then any finite dimensional $H$-invariant subspace $W \subseteq V$ is automatically G-invariant (and in fact G-fixed pointwise).

Proof. For a representation such as $\rho$, and $G$ minimally almost periodic, it is proved in [21] that if $v \in V$ is $H$-fixed, then it is $G$ fixed. The same argument applies to holomorphic representations of complex groups. Now the action $(g, T) \rightarrow \rho_{g} T \rho_{g}^{-1}$ is just such an action. If $T \in \mathscr{H} \mathscr{S}(V)$ and $\rho_{h} T=T \rho_{h}$ for all $h \in H$, then by the above we have $\rho_{g} T=T \rho_{g}$ for all $g \in G$, as required.

Now let $P_{W}$ be the orthogonal projection of $V$ on $W$. Since $W$ is finite dimensional, $P_{W} \in \mathscr{C} \mathscr{S}(V)$. By $H$-invariance and the fact that $\rho$ is unitary, $\rho_{h} P_{W}=P_{W} \rho_{h}$ for all $h \in H$. By the above, $\rho_{g} P_{W}=$ $P_{w} \rho_{g}$ for all $g \in G$, which means that $W$ is $G$-invariant. Clearly, it is $G$-fixed.

We remark that the extension of 6.3 to infinite dimensional subspaces $W$ is false. In general, the compact quotient case of 6.3 as well as the corresponding statement in [21] is also false.

\section{REFERENCES}

1. A. Borel, Linear Algebraic Groups, Benjamin, New York, 1969.

2. H. Furstenberg, A note on Borel's density theorem, Proc. Amer. Math. Soc., 55 (1976), 209-212.

3. M. Goto, Faithful representations of Lie groups I, Math. Japonicae, 1 (1948), 107-119.

4. —_, Faithful representations of Lie groups II, Nagoya Math. J., 1 (1950), 91-107.

5. F. Greenleaf, M. Moskowitz, and L. P. Rothschild, Unbounded conjugacy classes in Lie groups and location of central measures, Acta Math., 132 (1974), 225-243. 
F. Greenleaf, M. Moskowitz and L. P. Rothschild, Compactness of certain homogeneous spaces of finite volume, Amer. J. Math., 97 (1975), 248-259.

7. - Automorphisms, orbits, and homogeneous spaces of nonconnected Lie groups, Math. Annalen, 212 (1974), [45-155.

8. S. Grosser and M. Moskowitz, Compactness conditions in topological groups, I, II,

J. fur Reine und Angew. Math., 246 (1971), 1-40.

9. G. Hochschild, The structure of Lie groups, Holden-Day, San Francisco, 1965.

10. G. Hochschild, On representing analytic groups with their automorphisms, Pacific J. Math., 77 (1978), to appear.

11. K. Iwasawa, On some types of topological groups, Ann. of Math., 50 (1949), $507-558$.

12. G. W. Mackey, The theory of group representations, Univ. of Chicago Lecture Notes, Chicago, 1955.

13. M. Moskowitz, On the density theorems of Borel and Furstenberg, Arkiv för Mathematik, 16 (1978), 11-27.

14. — Automorphisms of bounded displacement and bounded cocycles, Monatshefte für Math., 85 (1978), to appear.

15. - Faithful representations and a local property of Lie groups, Math. Zeit., 143 (1975), 193-198.

16. C. C. Moore, Ergodicity of flows in homogeneous spaces, Amer. J. Math., 88 (1966), 154-178.

17. S. Rothman, The von Neumann kernel and minimally almost periodic groups, CUNY Graduate Center dissertation, 1978. Trans. Amer. Math. Soc., to appear.

18. J. Tits, Automorphismes de deplacement bornes des groupes de Lie, Topology, 31 (suppl. 1) (1964), 97-107.

19. S. P. Wang, On density properties of certain subgroups of locally compact groups, Duke Math. J., 43 (1976), 561-578.

20. - The dual spaces of semisimple Lie groups, Amer J. Math., 91 (1969), 921-937.

21. On isolated points in the dual spaces of locally compact groups, Math. Annalen, 219 (1975), 19-34.

22. H. Whitney, Elementary structure of real algebraic varieties, Annals of Math., 66 (1957), 545-556.

23. D. P. Zelobenko, Compact Lie groups and their representations, Translations of Math. Monographs, v. 40, American Math. Soc., Providence, 1973.

Received January 16, 1979. This research was supported in part by NSF Grants MCS 78-02153 and MCS 77-05201, respectively.

COURANT INSTITUTE

NeW YoRK UNIVERSITY

NeW YoRK, NY 10012

AND

Graduate Center

City University OF New YoRK

NEW YoRK, NY 10036 



\section{PACIFIC JOURNAL OF MATHEMATICS}

\section{EDITORS}

DONALD BABBITT (Managing Editor)

University of Galifornia

Los Angeles, California 90024

HUGo RossI

University of Utah

Salt Lake City, UT 84112

C. C. MOORE AND ANDREW OGG

University of California

Berkeley, CA 94720
J. DUGUNDJI

Department of Mathematics University of Southern California Los Angeles, California 90007

R. FINN AND J. MILGRAM Stanford University Stanford, California 94305

\section{ASSOCIATE EDITORS}

E. F. BECKENBACH

B. H. NEUMANN

F. WOLF

K. YosHIDA

\section{SUPPORTING INSTITUTIONS}

UNIVERSITY OF BRITISH COLUMBIA CALIFORNIA INSTITUTE OF TECHNOLOGY UNIVERSITY OF CALIFORNIA MONTANA STATE UNIVERSITY UNIVERSITY OF NEVADA, RENO NEW MEXICO STATE UNIVERSITY OREGON STATE UNIVERSITY UNIVERSITY OF OREGON
UNIVERSITY OF SOUTHERN CALIFONIA STANFORD UNIVERSITY UNIVERSITY OF HAWAII UNIVERSITY OF TOKYO UNIVERSITY OF UTAH WASHINGTON STATE UNIVERSITY UNIVERSITY OF WASHINGTON 


\section{Pacific Journal of Mathematics}

\section{Vol. 86, No. $1 \quad$ November, 1980}

Gert Einar Torsten Almkvist, Invariants, mostly old ones .............. 1

Hyman Bass, Groups of integral representation type ................ 15

A. Białynicki-Birula, On action of SL(2) on complete algebraic

varieties........................................

Frederick Paul Greenleaf and Martin Allen Moskowitz, Groups of

automorphisms of Lie groups: density properties, bounded orbits, and

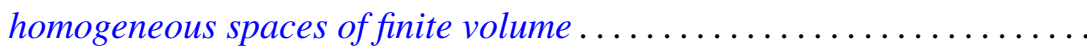

Raymond Taylor Hoobler, A cohomological interpretation of Brauer groups

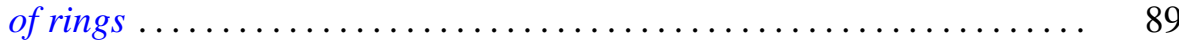

Irving Kaplansky, Superalgebras ........................ 93

Jerrold Lewis Kleinstein and Alex I. Rosenberg, Succinct and

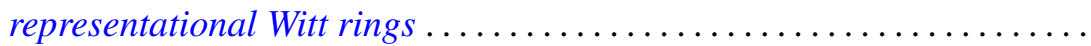

E. R. Kolchin, On universal extensions of differential fields ............ 139

Andy R. Magid, Analytic subgroups of affine algebraic groups. II ....... 145

Calvin Cooper Moore, The Mautner phenomenon for general unitary

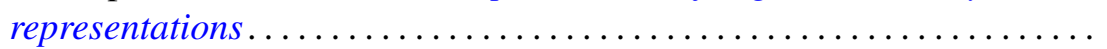

George Daniel Mostow, On a remarkable class of polyhedra in complex hyperbolic space ................................ 171

Brian Lee Peterson, Extensions of pro-affine algebraic groups. II . . . . . . 277

John Henry Reinoehl, Lie algebras and affine algebraic groups......... 287

Maxwell Alexander Rosenlicht, Differential valuations .

John Brendan Sullivan, The second Lie algebra cohomology group and Weyl modules..................................... 321

Moss Eisenberg Sweedler, Right derivations and right differential

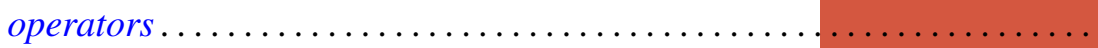

Bostwick Frampton Wyman, Time varying linear discrete-time systems. II. Duality.................................. 